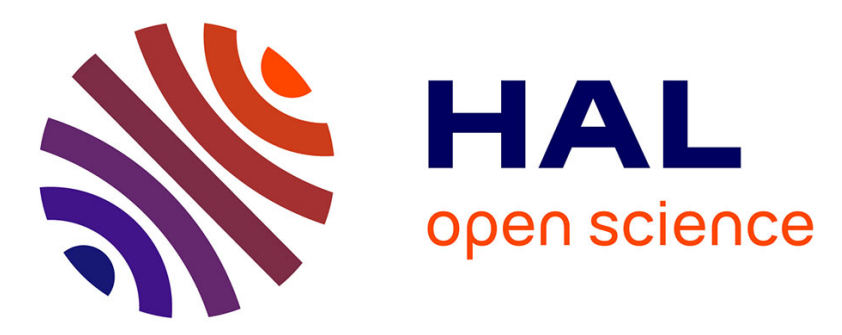

\title{
Generalized radiative transfer equation for porous medium upscaling: Application to the radiative Fourier law
}

J. Taine, Fabien Bellet, Vincent Leroy, Estelle Iacona

\section{- To cite this version:}

J. Taine, Fabien Bellet, Vincent Leroy, Estelle Iacona. Generalized radiative transfer equation for porous medium upscaling: Application to the radiative Fourier law. International Journal of Heat and Mass Transfer, 2010, 53 (19-20), pp.4071-4081. 10.1016/j.ijheatmasstransfer.2010.05.027 . hal00491742

\section{HAL Id: hal-00491742 \\ https://hal.science/hal-00491742}

Submitted on 6 Oct 2011

HAL is a multi-disciplinary open access archive for the deposit and dissemination of scientific research documents, whether they are published or not. The documents may come from teaching and research institutions in France or abroad, or from public or private research centers.
L'archive ouverte pluridisciplinaire HAL, est destinée au dépôt et à la diffusion de documents scientifiques de niveau recherche, publiés ou non, émanant des établissements d'enseignement et de recherche français ou étrangers, des laboratoires publics ou privés. 


\title{
Generalized radiative transfer equation for porous medium upscaling: application to the radiative Fourier law
}

\author{
Jean Taine ${ }^{\mathrm{a}, *}$, Fabien Bellet ${ }^{\mathrm{a}}$, Vincent Leroy $^{\mathrm{a}}$, Estelle Iacona $^{\mathrm{a}}$ \\ ${ }^{a}$ Laboratoire EM2C, École Centrale Paris - UPR 288 CNRS, Bâtiment Péclet, 92295 Châtenay-Malabry \\ Cedex, France
}

\begin{abstract}
Many porous media cannot be homogenized as Beerian semi transparent media. Effective extinction, absorption and scattering coefficients can indeed have no physical meaning for small or intermediate optical thicknesses. A Generalized Radiative Transfer Equation (GRTE), directly based on the extinction cumulative distribution function, the absorption and scattering cumulative probabilities and the scattering phase function is established for this optical thickness range. It can be solved by a statistical Monte Carlo approach. For a phase of a porous medium that is optically thick at local scale, the GRTE degenerates into a classical Beerian RTE. In these conditions, a radiative conductivity tensor is directly obtained, by a perturbation method, and expressed with the radiative coefficients of this RTE and temperature. As illustrations, exhaustive radiative conductivity results are given for a set of overlapping transparent spheres within an opaque phase and for opaque rod bundles.
\end{abstract}

Keywords:

Radiation, radiative conductivity, porous media, upscaling, statistical physics

\section{Nomenclature}

\section{Latin symbols}

$\mathrm{Kn}^{\text {ext }}$ Knudsen number related to radiative extinction

A Specific area per unit volume of the whole porous medium

E Extinction point

$g \quad$ Asymmetry factor of scattering

$G_{\text {ext }} \quad$ Extinction cumulative distribution function

\footnotetext{
${ }^{*}$ Corresponding author

Email address: jean.taine@ecp.fr (Jean Taine)
} 
$g_{\text {ext }} \quad$ Extinction cumulative distribution function for a Beerian medium

$k^{R} \quad$ Radiative conductivity

$M \quad$ Current point

$n \quad$ Refraction index

$P \quad$ Cumulative probability

$p \quad$ Cumulative probability for a Beerian medium

$p_{\nu} \quad$ Scattering phase function

$q_{i}^{R} \quad$ Radiative flux vector

$s, s^{\prime}, s_{1}, s_{1}^{\prime}$ Curvilinear abscissas along a ray

T Temperature

$u_{i} \quad$ Director cosine of axis $i$

$x_{i} \quad$ Coordinate of axis $i$

$S^{e} \quad$ Emission source term

$S^{s c} \quad$ Scattering source term

$S \quad$ Total source term

Greek symbols

$B \quad$ Generalized extinction coefficient at equilibrium

$K \quad$ Generalized absorption coefficient at equilibrium

$\alpha^{h} \quad$ Hemispherical interface absorptivity

$\beta \quad$ Extinction coefficient

$\kappa \quad$ Absorption coefficient

$\Omega \quad$ Solid angle

$\omega \quad$ Albedo

П $\quad$ Porosity

$\Sigma \quad$ Generalized scattering coefficient at equilibrium 


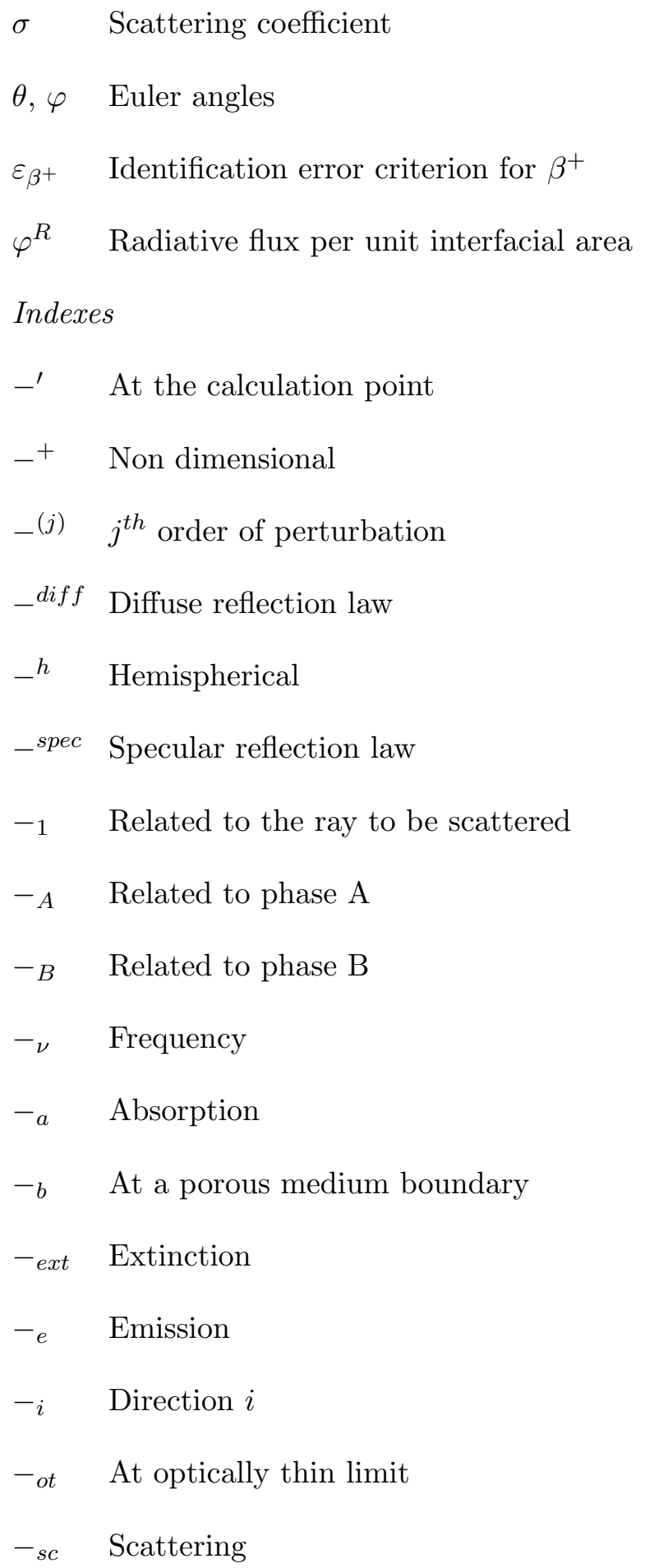




\section{Introduction}

Accurate radiative transfer models applied to porous media are required for energy technologies, for instance in ceramic foams for catalytic combustion, in porous materials of solid oxides fuel cells, in reticulated ceramics and packed beds used in solar absorbers and solar thermochemical reactors, in degraded rod bundles of a nuclear reactor core for the modeling of a severe accident... For all these applications, the key step is the accurate characterization of the radiative properties of the considered porous medium. This characterization is generally based on the non proved assumption that any porous material can be modeled by an equivalent semi transparent medium following Beer's laws, i.e. defined by extinction, absorption and scattering coefficients.

In the case of absorbing and scattering materials made of spheres or cylinders randomly oriented, some authors $[1,2]$ have simulated by a Monte Carlo technique a complete radiative transfer. They have defined the limits of validity of the independent scattering assumption, but only from the point of view of shadow effects.

The most popular method to characterize radiative properties is a parameter identification technique comparing experimental data, usually reflected or transmitted fluxes, and the predictions of a radiative transfer model based on the previous coefficients and on a phase function depending on general parameters $([3,4,5,6,7,8])$. A more detailed bibliography is given in [9] in the case of reticulated ceramics. These works are pertinent in so far as Beer's laws can be applied to the medium. Because of the large number of parameters to determine, the parameter identification technique practically always leads

to a solution, but does not allow to physically validate the set of parameters that is obtained.

Using a deterministic mechanical approach, Consalvi et al. [10] have generalized to radiative transfer the homogenization technique developed by Whitaker [11] and Quintard [12] for mass transfer or other heat transfer modes. This method has then been developed by Lipinski et al. [13]. It is mainly based on mathematical theorems, and the specific features of radiation are only taken into account by a radiative transfer equation and boundary conditions, associated to any phase of the porous medium at local scale. The radiation modeling is degraded, by a spatial and directional averaging technique, to the scale of homogenization of the material system. It seems difficult, in this approach, to account for the characteristic scales of radiation, which can be smaller than the arbitrary chosen homogenization scale, and for spectral correlations in a gaseous phase.

A new statistical approach, called Radiative Distribution Function Identification (RDFI), has been developed since 2004. Its aim is to directly characterize the equivalent radiative properties of a porous medium, with a spatial resolution $d$, in so far as both the morphology of the medium and its radiative properties at local scale, i.e. lower 
than $d$, are known. In these conditions, a homogenized phase of a porous medium is exhaustively characterized by statistical functions: the extinction cumulative distribution function, the absorption cumulative probability or the scattering one and the scattering phase function with sometimes an associated effective refractive optical index. These statistical functions have been determined by an original Monte Carlo technique, which strongly differs from the Monte Carlo approach previously cited or sometimes used in parameter identification techniques. Indeed, there is no simulation of radiative transfer in this technique, but a direct characterization of the radiative properties of the medium from their statistical definitions. This approach has been first developed by Tancrez and Taine [14], while applied to a statistically isotropic and homogeneous porous medium. This virtual medium is made of sets of overlapping spheres with a dispersed radius distribution (for instance Gaussian), either opaque within a transparent phase (DOOS) or transparent within an opaque phase (DOTS). These last media are close to ceramic foams with an opaque solid phase. An important interest of the RDFI approach is to include a quantitative validation criterion of the assumption that the equivalent medium follows Beer's laws. This property is, for instance, not true in the whole optical thickness range in the case of a DOTS medium of porosity less than about 0.6. Zeghondy et al. $[15,16]$ have generalized the previous model to a statistically anisotropic real porous medium, a mullite foam of porosity close to 0.85 , in a spectral range such as the solid phase is semi transparent at local scale. In this case, anisotropic extinction, absorption and scattering coefficients have been accurately determined and physically validated. The phase function has been directly determined. Petrasch et al. [17], Haussener et al. [18, 19] have applied the same method to determine the Beerian radiative properties of high porosity reticulated ceramics and packed beds used in solar absorbers and solar thermochemical reactors. Bellet et al. [20] have characterized, with the RDFI method, bundles of opaque rods of an intact nuclear core, which is a strongly anisotropic porous medium. The authors have shown, in this case, that an effective Beerian semi transparent medium is not an accurate representation. An effective radiative conductivity, related to transfer in the plane perpendicular to the rods, has also been determined for this medium from a perturbation method of the radiative transfer equation. Gusarov [21] has developed a similar perturbation method to calculate a radiative conductivity.

Sec.2 deals with a general characterization of the radiative properties of porous media that do not follow Beer's laws, for instance intermediate and low porosity foams, intact or degraded rod bundles... In Sec.3, a generalized radiative transfer equation, directly based on the radiative statistical distribution functions, is established. Sec.4 is devoted to the direct determination of a radiative conductivity tensor, associated with a radiative diffusion model, for a medium that is optically thick at local scale. Two application examples are treated in Sec.5, dealing with a set of overlapping spheres within an opaque 
phase and with rod bundles. A conclusion is finally drawn in Sec.6.

\section{General characterization of a porous medium}

All the porous media that are studied here are assumed to be statistically uniform.

In the upscaling approach to follow, any non opaque phase of a porous medium, even transparent, is homogenized by introducing an effective continuous semi transparent medium, characterized by effective radiative properties. This effective semi transparent medium may follow Beer's laws, and effective extinction, absorption and scattering coefficients are then introduced. If it does not follow Beer's laws and is therefore a non Beerian effective semi transparent medium, a more general characterization is developed.

In the case of a porous medium with two phases that are respectively transparent and opaque at local scale, a semi transparent medium, characterized by effective radiative properties, is associated with the radiative transfer between the opaque interfaces [14]. In the case of a porous medium with semi transparent phases at local scale, effective radiative properties are also associated with each homogenized phase, and strongly differ from the radiative properties at local scale [15].

In any case, the radiative effective properties of a homogenized phase of a porous medium, possibly statistically anisotropic, are completely characterized by four statistical functions, defined in the whole system of coordinates [20]:

i) An extinction cumulative distribution function $G_{\text {ext } \nu}\left(s^{\prime}-s, \theta, \varphi\right)$, which is in fact the cumulative distribution function of the lengths $s^{\prime}-s$ of all the intervals $M E$ joining, in the direction defined by $(\theta, \varphi)$, any current point $M$ of abscissa $s$ within the considered phase to the associated extinction point $E$ of abscissa $s^{\prime}$. $E$ can be within the phase, if this phase is semi transparent at local scale, or, in any case, an impact point at the interface with another phase. It is worth noticing that $1-G_{\text {ext } \nu}\left(s^{\prime}-s, \theta, \varphi\right)=\tau_{\nu}\left(s^{\prime}-s, \theta, \varphi\right)$, which is the transmissivity of the homogenized phase in the direction $(\theta, \varphi)$.

ii) An absorption cumulative probability $P_{a \nu}\left(s^{\prime}-s, \theta, \varphi\right)$, which is the probability that a ray issued from any point $M(s)$ of a phase in the direction $(\theta, \varphi)$ is absorbed within this phase or at an opaque interface before a distance $s^{\prime}-s$ from $M$,

or, which is equivalent, a scattering cumulative probability $P_{s c \nu}\left(s^{\prime}-s, \theta, \varphi\right)$, which is the probability that a ray issued from any point $M(s)$ of a phase in the direction $(\theta, \varphi)$ is scattered within this phase or at an interface before a distance $s^{\prime}-s$ from $M$ [15].

These first statistical functions obviously verify:

$$
G_{\text {ext } \nu}\left(s^{\prime}-s, \theta, \varphi\right)=P_{a \nu}\left(s^{\prime}-s, \theta, \varphi\right)+P_{s c \nu}\left(s^{\prime}-s, \theta, \varphi\right)
$$

iii) A phase function $p_{\nu}\left(\theta, \varphi, \theta^{\prime}, \varphi^{\prime}\right)$, depending in the general case on the directions of 
both incident and scattered rays, respectively characterized by $(\theta, \varphi)$ and $\left(\theta^{\prime}, \varphi^{\prime}\right)$.

iv) An effective refractive index $n_{\nu}(\theta, \varphi)$, in so far as $P_{s c \nu}\left(s^{\prime}-s, \theta, \varphi\right)$ and the other statistical functions depend on the direction $(\theta, \varphi)[20]$. The determination of $n_{\nu}(\theta, \varphi)$ is detailed in Appendix A.

These four independent statistical functions can be determined from the knowledge of both the real morphology of the porous medium, obtained for instance from an $\mathrm{X}$ ray $[15,17,18]$ or $\gamma$ ray [22] tomography, and the radiative properties of the medium at local scale, i.e. at a spatial scale that is not taken into account by the tomography.

A statistical Monte Carlo approach is used for the direct determination of $G_{\text {ext } \nu}$, $P_{s c \nu}$ and $p_{\nu}$ for a porous medium with opaque and transparent phases at local scale $[14,17,18,20]$, opaque and semi transparent phases, or semi transparent phases at local scale [15]. It is based on a huge number of rays that are shot from randomly points within the propagation phase, in randomly chosen directions covering the whole space. Two key conditions have to be carefully fulfilled:

i) The shooting zone must be representative of the whole porous medium (see an example in Figs.1(a) and 1(b) for intact opaque rod bundles in triangular and square configurations). The shooting zone is defined in agreement with the possible symmetries and periodicities of the porous medium, case for instance of [20]. In porous media such as foams, the statistical uniformity needs to be checked (see for instance [15]).

[Figure 1 about here.]

ii) All rays must be followed until their extinction (if it eventually happens).

Under these conditions, the radiative statistical functions that are obtained are representative of the radiative properties of the whole homogenized phase over the whole optical thickness range, in the case of a statistically uniform medium.

In many cases, as discussed in the following, the homogenized phase can be considered as Beerian. In these conditions, the extinction cumulative distribution function, called $g_{\text {ext } \nu}\left(s^{\prime}-s, \theta, \varphi\right)$, and the absorption and scattering cumulative probabilities, called $P_{a \nu}\left(s^{\prime}-s, \theta, \varphi\right)$ and $P_{s c \nu}\left(s^{\prime}-s, \theta, \varphi\right)$, are simply given by

$$
\begin{aligned}
g_{\text {ext } \nu}\left(s^{\prime}-s, \theta, \varphi\right) & =1-\exp \left[-\left(s^{\prime}-s\right) \beta_{\nu}(\theta, \varphi)\right], \\
P_{a \nu}\left(s^{\prime}-s, \theta, \varphi\right)=1-P_{s c \nu}\left(s^{\prime}-s, \theta, \varphi\right) & =\left[1-\omega_{\nu}(\theta, \varphi)\right]\left(1-\exp \left[-\left(s^{\prime}-s\right) \beta_{\nu}(\theta, \varphi)\right]\right),
\end{aligned}
$$

where the medium extinction coefficient $\beta_{\nu}(\theta, \varphi)$, scattering coefficient $\sigma_{\nu}(\theta, \varphi)$ and albedo $\omega_{\nu}(\theta, \varphi)$, equal to $\sigma_{\nu}(\theta, \varphi) / \beta_{\nu}(\theta, \varphi)$, are introduced.

But all real porous media cannot be accurately modeled by equivalent Beerian semi transparent media. In the following examples, the statistical function $G_{\text {ext }}$, and consequently $P_{s c}$, are accurately determined with a typical relative error (standard deviation) 
smaller than $10^{-4}$.

The first example is given in Fig.2, which deals with a set of Dispersed radius Overlapping Transparent Spheres (DOTS) within an opaque medium.

[Figure 2 about here.]

It is a realistic model for a foam that is statistically isotropic. The quantity $\ln \left(1-G_{\text {ext }}\right)$ is expressed versus a non dimensional distance $s^{+}=\beta_{O T}\left(s^{\prime}-s\right)$, where $\beta_{O T}$ is the extinction coefficient at the limit of an optically thin medium (see [14]). For statistically isotropic media, $\beta_{O T}$ is equal to $A /(4 \Pi)$, where $A$ is the interfacial area per unit volume of the porous medium and $\Pi$ its porosity. It appears that $G_{\text {ext }}$ cannot be modeled by an exponential function in the optical thickness range $[x, 2]$ when the porosity is less than 0.6 . The initial value $x$ strongly depends on the porosity. This figure is also discussed in Sec.5.1.

Another example, shown in Fig.3, is related to triangular and square configurations of intact opaque rod bundles, which is a statistically anisotropic system encountered for instance in a nuclear reactor core.

[Figure 3 about here.]

The $G_{\text {ext }}$ field strongly differs from the exponential functions associated with the Beerian semi transparent model. This figure is also discussed in Sec.5.2.

\section{Generalized Radiative Transfer Equation}

A generalized formulation of the Radiative Transfer Equation (RTE), directly based on the statistical functions $G_{e x t}, P_{a \nu}, P_{s c \nu}, p_{\nu}$ and the effective refractive index $n_{\nu}$ is required when the three first statistical functions are not exponential. The following assumptions are made:

i) For the sake of simplicity, the possible dependence on the current direction $(\theta, \varphi)$ of the statistical functions is omitted in this part. All the equations of Secs. 3 to 4 are nevertheless still valid when $G_{e x t}, P_{a \nu}, P_{s c \nu}, n_{\nu}$ depend on $(\theta, \varphi)$.

ii) The statistical functions are spatially uniform in the homogenized phase: for example, the transmissivity of the medium from $s$ to $s^{\prime}$ in the direction $(\theta, \varphi), 1-G_{\text {ext } \nu}$, only depends on the distance $v=s^{\prime}-s$.

iii) Only the core of the porous medium is considered: if $s^{\prime}-s_{b}$ is the distance to the closest boundary of the whole porous medium, $G_{\text {ext }}\left(s^{\prime}-s_{b}\right)$ is assumed to be close to 1 . A specific model is required in the radiative boundary layer, i.e. such as $G_{e x t \nu}\left(s^{\prime}-s_{b}\right)<1$, but it is still an open research field.

iv) All the radiation wavelengths are small compared to the typical pore size: diffraction phenomena can then be neglected, and the laws of geometrical optics are valid. 


\subsection{Physical meaning and general expression of intensity}

The emission and scattering phenomena, which occur around a point $M$, within an elementary cylinder of cross section $\mathrm{d} S$ and length $\mathrm{d} s$, in an elementary solid angle $\mathrm{d} \Omega$ around the direction $(\theta, \varphi)$, generate a radiative flux in this direction: $S_{\nu}(s, \theta, \varphi) \mathrm{d} s \mathrm{~d} S \mathrm{~d} \Omega \mathrm{d} \nu$. In this expression, $S_{\nu}(s, \theta, \varphi)$ is the total source term in the direction $(\theta, \varphi)$, within the volume $\mathrm{d} S \mathrm{~d} s$ characterized by a temperature $T(s)$. It includes emission and scattering phenomena, i.e.:

$$
S_{\nu}(s, \theta, \varphi)=S_{e \nu}[T(s)]+S_{s c \nu}(s, \theta, \varphi) .
$$

Consequently, an intensity $S_{\nu}(s, \theta, \varphi) \mathrm{d} s$, associated to this flux, is generated at the source point $M$, in the direction $(\theta, \varphi)$, around the abscissa $s$. For a non Beerian homogenized phase, the radiative flux at a point $M^{\prime}$ of abscissa $s^{\prime}$ results from the cumulated effect of the elementary fluxes due to all the source terms from $s_{b}$, abscissa of an arbitrary far boundary of the whole porous medium, to $s^{\prime}$, and transmitted up to $s^{\prime}$. The source point $M$ belongs, at local scale, to an opaque interface (medium with opaque and transparent phases) or to the volume of the phase (medium with semi transparent phases), but only to a continuum after homogenization. In any case, the transmissivity in the direction $(\theta, \varphi)$ between $M(s)$ and a current point of the homogenized phase $M^{\prime}\left(s^{\prime}\right)$ is then given by $1-G_{\text {ext } \nu}\left(s^{\prime}-s\right)$ (see Sec.2). The intensity associated with this flux, at the abscissa $s^{\prime}$ in the direction $(\theta, \varphi)$, is then:

$$
I_{\nu}\left(s^{\prime}, \theta, \varphi\right)=\int_{s_{b}}^{s^{\prime}} S_{\nu}(s, \theta, \varphi) \tau_{\nu}\left(s^{\prime}-s\right) \mathrm{d} s=\int_{s_{b}}^{s^{\prime}} S_{\nu}(s, \theta, \varphi)\left[1-G_{e x t \nu}\left(s^{\prime}-s\right)\right] \mathrm{d} s
$$

The physical meaning of $I_{\nu}\left(s^{\prime}, \theta, \varphi\right)$ must be emphasized. This intensity is only defined in the virtual continuous homogenized phase, which is the result of this type of statistical modeling [14]. This statistical homogenization of the radiation field in a phase completely differs from the common deterministic homogenization techniques of the material system that some authors have applied to radiation [10, 13]. But these two independent homogenization techniques are applied around the same current points of the real porous medium.

The radiative approach described here is statistical. In an arbitrarily small volume of the whole homogenized porous medium $\mathrm{d} V$, any homogenized phase is only characterized by a probability of presence, $\Pi$ for a fluid phase, commonly called porosity, and $1-\Pi$ for the solid phase. Indeed, there is no defined volume associated with any homogenized phase. Consequently, the intensity $I_{\nu}\left(s^{\prime}, \theta, \varphi\right)$ associated with the homogenized phase is continuous and, by convention, defined in $\mathrm{d} V$, elementary volume element of the whole porous medium. Consequently, it is, by convention, proportional to the porosity $\Pi$ associated with the real phase. The porosity will be introduced in emission source terms only, 
as we will see later.

As there is no theoretical spatial limitation to the determination of the radiative statistical functions $G_{e x t}, P_{s c \nu}$, and $p_{\nu}$ (see Sec.2), the most important spatial limitations encountered in the calculation of the intensity with Eq.4 are due to the temperature field. In practice, the temperature field is calculated from the energy balance equation of the homogenized material system.

The variations in the elementary range $\left[s^{\prime}, s^{\prime}+\mathrm{d} s^{\prime}\right]$ of the intensity associated with extinction, absorption and scattering phenomena, called $\mathrm{d} I_{\text {ext } \nu}\left(s^{\prime}, \theta, \varphi\right), \mathrm{d} I_{a \nu}\left(s^{\prime}, \theta, \varphi\right)$ and $\mathrm{d} I_{s c \nu}\left(s^{\prime}, \theta, \varphi\right)$ respectively, are given by:

$$
\mathrm{d} I_{c, \nu}\left(s^{\prime}, \theta, \varphi\right)=-\int_{s_{b}}^{s^{\prime}} S_{\nu}(s, \theta, \varphi) \mathrm{d}\left[X_{c \nu}\left(s^{\prime}-s\right)\right] \mathrm{d} s \quad \text { with } \quad c=e x t, a \text { or } s c
$$

where $X_{c \nu}$ represents $G_{e x t \nu}, P_{a \nu}$ or $P_{s c \nu}$, and d $\left[X_{c \nu}\left(s^{\prime}-s\right)\right]$ is equal to $X_{c \nu}\left(\left[s^{\prime}-s\right]+\right.$ $\left.\mathrm{d} s^{\prime}\right)-X_{c \nu}\left(s^{\prime}-s\right)=\frac{\mathrm{d} X_{c \nu}}{\mathrm{d} v}\left(s^{\prime}-s,\right) \mathrm{d} s^{\prime}$. Indeed, in the case of extinction, for instance, the variation of intensity due to extinction between $s^{\prime}$ and $s^{\prime}+\mathrm{d} s^{\prime}$ is the difference between intensities at $s^{\prime}$ and $s^{\prime}+\mathrm{d} s^{\prime}$, to which is added the intensity that is generated by all the source terms in $\left[s^{\prime}, s^{\prime}+\mathrm{d} s^{\prime}\right]$.

\subsection{Thermal equilibrium of an optically thick phase}

In thermal equilibrium conditions at temperature $T$, the total source term $S_{\nu}^{\circ}$ is spatially uniform. The intensity $n_{\nu}^{2} \Pi I_{\nu}^{\circ}(T)$ is also uniform; $\Pi$ is the porosity of the phase in the whole porous medium and $\Pi I_{\nu}^{\circ}(T)$ is the equilibrium intensity in vacuum. Consequently, if we assume that the phase is optically thick from $s_{b}$ to $s^{\prime}$, i.e $G_{\text {ext }}\left(s^{\prime}-s_{b}\right)$ is close to 1 , the expression of $S_{\nu}^{\circ}$ is deduced from Eq.4:

$$
S_{\nu}^{\circ}=\frac{n_{\nu}^{2} \Pi I_{\nu}^{\circ}(T)}{\int_{0}^{\infty}\left[1-G_{e x t \nu}(v,)\right] \mathrm{d} v}=B_{\nu} n_{\nu}^{2} \Pi I_{\nu}^{\circ}(T)
$$

where $v=s^{\prime}-s$ is a dummy variable. This equation introduces $B_{\nu}$, a generalized extinction coefficient at equilibrium:

$$
B_{\nu}=\left(\int_{0}^{\infty}\left[1-G_{e x t \nu}(v)\right] \mathrm{d} v\right)^{-1} .
$$

Eq.5 applied to scattering phenomena becomes, after some mathematical manipulations involving $v=s^{\prime}-s$ :

$$
\begin{aligned}
{\left[\mathrm{d} I_{s c \nu}\right]^{\circ}\left(s^{\prime}\right) } & =-P_{s c \nu}(\infty) B_{\nu} n_{\nu}^{2} \Pi I_{\nu}^{\circ}(T) \mathrm{d} s^{\prime} \\
& =-\Sigma_{\nu} n_{\nu}^{2} \Pi I_{\nu}^{\circ}(T) \mathrm{d} s^{\prime}
\end{aligned}
$$


which allows us to introduce the generalized scattering coefficient at equilibrium $\Sigma_{\nu}$, equal to $P_{s c \nu}(\infty) B_{\nu}$, and the corresponding albedo $P_{s c \nu}(\infty)$. In a similar manner, the absorbed intensity at equilibrium in the range $\left[s^{\prime}, s^{\prime}+\mathrm{d} s^{\prime}\right]$ can be written, using Eqs.1 and 5 :

$$
\begin{aligned}
{\left[\mathrm{d} I_{a \nu}\right]^{\circ}\left(s^{\prime}\right) } & =-\left[1-P_{s c \nu}(\infty)\right] B_{\nu} n_{\nu}^{2}\left(\Pi I_{\nu}^{\circ}(T) \mathrm{d} s^{\prime}\right. \\
& =-K_{\nu} n_{\nu}^{2} \Pi I_{\nu}^{\circ}(T) \mathrm{d} s^{\prime}
\end{aligned}
$$

which allows us to introduce the generalized absorption coefficient at equilibrium $K_{\nu}$, equal to $\left[1-P_{s c \nu}(\infty)\right] B_{\nu}$.

In an application involving radiative transfer, neither the radiation field nor the material system are in thermal equilibrium conditions. But, at local scale, the material system is generally considered in Local Thermal Equilibrium (LTE) [23, 24], i.e. an arbitrary small volume element $\mathrm{d} V$ can be characterized by a temperature $T$ during an arbitrary short duration $\mathrm{d} t$. In so far as the thermal emission is a property of the material system, the emission source term per unit volume, in LTE conditions at temperature $T$, is equal to the absorption term:

$$
S_{e \nu}(T)=K_{\nu} n_{\nu}^{2}\left(\Pi I_{\nu}^{\circ}(T)\right.
$$

\subsection{Generalized Radiative Transfer Equation}

The Generalized Radiative Transfer Equation (GRTE) expresses the variation of the intensity $I_{\nu}\left(s^{\prime}, \theta, \varphi\right)$ at $M^{\prime}\left(s^{\prime}\right)$ along the direction $(\theta, \varphi)$ :

$$
\frac{\mathrm{d} I_{\nu}}{\mathrm{d} s^{\prime}}\left(s^{\prime}, \theta, \varphi\right)=-\frac{\mathrm{d} I_{e x t \nu}}{\mathrm{d} s^{\prime}}\left(s^{\prime}, \theta, \varphi\right)+S_{\nu}\left(s^{\prime}, \theta, \varphi\right)
$$

or, using Eq.5:

$$
\frac{\mathrm{d} I_{\nu}}{\mathrm{d} s^{\prime}}\left(s^{\prime}, \theta, \varphi\right)=-\int_{s_{b}}^{s^{\prime}} S_{\nu}(s, \theta, \varphi) \frac{\mathrm{d} G_{e x t \nu}}{\mathrm{d} v}\left(s^{\prime}-s\right) \mathrm{d} s+S_{\nu}\left(s^{\prime}, \theta, \varphi\right) .
$$

In $S_{\nu}\left(s^{\prime}, \theta, \varphi\right)$, the emission source term $S_{\nu}^{e}\left[T\left(s^{\prime}\right)\right]$ is given by Eq.10.

The main difficulty now is to express the scattering source term $S_{s c \nu}\left(s^{\prime}, \theta, \varphi\right)$. For the sake of simplicity, the study is limited here to a porous medium with two phases, one opaque and one transparent at local scale. Let us consider an elementary volume $\mathrm{d} V$ of the homogenized porous medium, around the point $M^{\prime}$ of abscissas $s^{\prime}$ and $s_{1}^{\prime}$ in the directions $(\theta, \varphi)$ and $\left(\theta_{1}, \varphi_{1}\right)$ respectively. Let $\mathrm{d} S^{\prime}$ and $\mathrm{d} S_{1}$ be the elementary sections of this volume, respectively normal to the directions $(\theta, \varphi)$ and $\left(\theta_{1}, \varphi_{1}\right)$, as shown in Figs.4. In our differential analysis, this volume $\mathrm{d} V$ is both equal to $\mathrm{d} S_{1} \mathrm{~d} s_{1}^{\prime}$ and $\mathrm{d} S^{\prime} \mathrm{d} s^{\prime}$.

[Figure 4 about here.] 
The power $\mathrm{d} \mathcal{P}_{\nu}\left(s_{1}, \theta_{1}, \varphi_{1}\right)$ issued from the homogenized phase belonging to an elementary volume $\mathrm{d} S_{1} \mathrm{~d} s_{1}$ between $s_{1}$ and $s_{1}+\mathrm{d} s_{1}$, in the elementary solid angle $\mathrm{d} \Omega_{1}$ around the direction $\left(\theta_{1}, \varphi_{1}\right)$, towards the elementary cross section $\mathrm{d} S_{1}$ of the control volume $\mathrm{d} V$ (see Fig.4(a)), is given by:

$$
\frac{\mathrm{d} \mathcal{P}_{\nu}\left(s_{1}, \theta_{1}, \varphi_{1}\right)}{\mathrm{d} S_{1} \mathrm{~d} s_{1} \mathrm{~d} \Omega_{1} \mathrm{~d} \nu}=S_{e \nu}\left[T\left(s_{1}\right)\right]+S_{s c \nu}\left(s_{1}, \theta_{1}, \varphi_{1}\right)=S_{\nu}\left(s_{1}, \theta_{1}, \varphi_{1}\right)
$$

The probability for this power to be scattered in the control volume $\mathrm{d} V$ around $M^{\prime}$, between the abscissa $s_{1}^{\prime}$ and $s_{1}^{\prime}+\mathrm{d} s_{1}^{\prime}$, in the elementary solid angle $\mathrm{d} \Omega$ around the direction $(\theta, \varphi)$, is $\mathrm{d}\left[P_{s c \nu}\left(s_{1}^{\prime}-s_{1}\right)\right]\left[p_{\nu}\left(\theta_{1}, \varphi_{1}, \theta, \varphi\right) \frac{\mathrm{d} \Omega}{4 \pi}\right]$. Consequently, the part $\mathrm{d} \mathcal{P}_{s c \nu}\left(s_{1}, s^{\prime}, \theta_{1}, \varphi_{1}, \theta, \varphi\right)$ of $\mathrm{d} \mathcal{P}_{\nu}\left(s_{1}, \theta_{1}, \varphi_{1}\right)$ that is scattered in the direction $(\theta, \varphi)$ by the volume $\mathrm{d} V$ is:

$$
\begin{aligned}
& \mathrm{d} \mathcal{P}_{s c \nu}\left(s_{1}, s^{\prime}, \theta_{1}, \varphi_{1}, \theta, \varphi\right) \\
= & {\left[\frac{\mathrm{d} P_{s c \nu}}{\mathrm{d} v}\left(s_{1}^{\prime}-s_{1}\right) \mathrm{d} s_{1}^{\prime}\right]\left[p_{\nu}\left(\theta_{1}, \varphi_{1}, \theta, \varphi\right) \frac{\mathrm{d} \Omega}{4 \pi}\right] S_{\nu}\left(s_{1}, \theta_{1}, \varphi_{1}\right) \mathrm{d} S_{1} \mathrm{~d} s_{1} \mathrm{~d} \Omega_{1} \mathrm{~d} \nu . }
\end{aligned}
$$

In this last equation, $\mathrm{d} S_{1} \mathrm{~d} s_{1}^{\prime}$ is equal to $\mathrm{d} V$. The scattering source term $S_{s c \nu}\left(s^{\prime}, \theta, \varphi\right)$ at $M^{\prime}$ of abscissa $s^{\prime}$ in the direction $(\theta, \varphi)$ is obtained by summing $\mathrm{d} \mathcal{P}_{s c \nu}\left(s_{1}, s^{\prime}, \theta_{1}, \varphi_{1}, \theta, \varphi\right)$ over both the solid angle $\mathrm{d} \Omega_{1}$ and $s_{1}$, and dividing by $\mathrm{d} V \mathrm{~d} \Omega \mathrm{d} \nu$, i.e.:

$$
S_{s c \nu}\left(s^{\prime}, \theta, \varphi\right)=\int_{4 \pi} \int_{s_{1 b}}^{s_{1}^{\prime}} \frac{\mathrm{d} P_{s c \nu}}{\mathrm{d} v}\left(s_{1}^{\prime}-s_{1}\right) \frac{p_{\nu}\left(\theta_{1}, \varphi_{1}, \theta, \varphi\right)}{4 \pi} S_{\nu}\left(s_{1}, \theta_{1}, \varphi_{1}\right) \mathrm{d} s_{1} \mathrm{~d} \Omega_{1} .
$$

It is worth noticing that in Eq.15, $s_{1}^{\prime}$ is the abscissa of the point $M^{\prime}$ in the current direction $\left(\theta_{1}, \varphi_{1}\right)$. In fact, the result depends on $s^{\prime}$, abscissa of $M^{\prime}$ in the direction $(\theta, \varphi)$.

Finally, the strongly implicit Generalized Radiative Transfer Equation (GRTE) associated with the homogenized phase is defined by Eq.12, in which are introduced $S_{e \nu}$ and $S_{s c \nu}$ issued from Eqs.10 and 15. In this generalized approach, the knowledge of the intensity at a point $s^{\prime}$ is not sufficient to compute the intensity variation in the range $\left[s^{\prime}, s^{\prime}+\mathrm{d} s^{\prime}\right]$, and consequently the radiative transfer in the system, contrary to the case of a semi transparent medium following Beer's laws. Indeed, the variation of the intensity $I_{\nu}\left(s^{\prime}, \theta, \varphi\right)$ at a given point and in a given direction explicitly depends on the extinction cumulative distribution function field along the considered direction, and moreover on the scattering cumulative probability at any point of the medium and in any direction.

\subsection{Practical use of the GRTE applied to a homogenized phase}

We discuss here the general case of porous media in non equilibrium, such as each phase is characterized by a specific temperature. Moreover, the temperature of an opaque interface between the phases a priori differs from the previous ones. As seen in Sec.3.1, 
the intensity can be defined for a homogenized phase within any volume element $\mathrm{d} V$ of a porous medium around a point $M$. Its spatial evolution is given by the associated GRTE, which depends:

i) on the statistical functions $G_{\text {ext } \nu}, P_{s c \nu}$ and $p_{\nu}$, which can been calculated without practical limitation related to the spatial resolution of the homogenization of the radiation field within the phase (see Sec.2),

ii) on emission source terms considered in LTE conditions of the material system. The spatial resolution of these emission source terms is, in practice, limited by the resolution on the temperature field within the phase, issued from the homogenization of the material system. Indeed, an emission source term characterizes the state of the material system.

From a practical point of view, the radiative vector $q_{i}^{R}\left(x_{j}\right)$ at a point $M$, of coordinates $x_{j}$ in tensorial notations, is obtained from the intensity field in $M$ by:

$$
q_{i}^{R}\left(x_{j}\right)=\int_{0}^{\infty} \int_{0}^{2 \pi} \int_{0}^{\pi} I_{\nu}\left(x_{j}, \theta, \varphi\right) u_{i} \sin \theta \mathrm{d} \theta \mathrm{d} \varphi \mathrm{d} \nu
$$

where $u_{i}$ is the unit vector associated with the direction $i$. Let us recall that, with the convention introduced in Sec.3.1, the porosity of the phase is implicitly taken into account in the expression of the intensity. The radiative power per unit volume $P^{R}\left(x_{j}\right)$ is deduced at any point $\mathrm{M}$ of the phase by:

$$
P^{R}\left(x_{j}\right)=\frac{\partial q_{i}^{R}}{\partial x_{i}}\left(x_{j}\right)
$$

$P^{R}\left(x_{j}\right)$ is a source term in the energy balance equation related to the phase material system, issued from its homogenization procedure. In the case of opaque and transparent phases at local scale, the homogenized phase is a virtual semi transparent medium which, in fact, represents random opaque interface elements. A more physical quantity, characterizing the coupling between the radiation field and the material system, is then the radiative flux per unit interfacial area $\varphi^{R}\left(x_{j}\right)$, simply given by:

$$
\varphi^{R}\left(x_{j}\right)=\frac{P^{R}\left(x_{j}\right)}{A}
$$

where $A$ is the interfacial area per unit volume of the whole porous medium. It is worth noticing that, in Eq.18, $P^{R}$ is proportional to the porosity $\Pi$. Consequently, $\varphi^{R}$ is proportional to the inverse of the interfacial area per unit volume of the transparent fluid phase. Finally, the deterministic mechanical homogenization method and the statistical radiative one are compatible and can be used in an iterative scheme. The temperature field, issued from the energy balance equation of the homogenized material system, allows us to calculate the intensity field at any point of a homogenized phase, and consequently 
$P^{R}$ and $\varphi^{R}$, which are source terms in the energy balance equation and also appear in the boundary conditions of the material system. This iterative treatment is similar to the treatment used in the modeling of systems in which turbulent combustion and radiation are coupled. The previous model can be applied with no difference to porous media with interconnected or closed pores. Nevertheless, this last characteristics of the medium has to be taken into account in the modeling of the material system.

The only practical way to solve the extremely implicit GRTE is a Monte Carlo approach, based on a statistical modeling of all the emission source terms within the system. Emission is modeled by shooting a huge number of radiative power quanta. All these quanta are subjected, along all their paths through the whole system, to a large number of scattering phenomena, stochastically and directly modeled by $P_{s c \nu}$. These quanta are also continuously extinguished by absorption, stochastically and directly modeled by $P_{a \nu}$. Indeed, the main interest of the Monte Carlo method is to be based on cumulative distribution functions or cumulative probabilities, which are here directly determined from the model.

\section{Limit of a locally optically thick porous medium}

A phase of a porous medium is often optically thick at a spatial scale $\delta$ such as it can be considered practically isothermal. Examples of these media are given at the end of Sec.2. It is established in Sec.4.1 that, in these conditions, the GRTE associated with a homogenized phase degenerates in a classical RTE. Consequently, the perturbation approach developed in [20], which allows us to simply characterize a Beerian homogenized phase by a radiative conductivity from a perturbation method applied to a classical RTE, can be applied. The corresponding Fourier law is introduced in Sec.4.2.

\subsection{Asymptotic expression of the GRTE}

Let us consider a homogenized phase that is optically thick at a spatial scale $\delta$ such as it can be considered as practically isothermal. Both the total source term $S_{\nu}\left(s^{\prime}, \theta, \varphi\right)$ and the intensity $I_{\nu}\left(s^{\prime}, \theta, \varphi\right)$ are uniform, for given direction and frequency, within a volume element of size $\delta$. From Eq.4, they are linked by

$$
S_{\nu}\left(s^{\prime}, \theta, \varphi\right)=I_{\nu}\left(s^{\prime}, \theta, \varphi\right)\left(\int_{s^{\prime}-\delta}^{s^{\prime}}\left[1-G_{e x t}\left(s^{\prime}-s\right)\right] \mathrm{d} s\right)^{-1}
$$

It is worth noticing that in Eq.19, the variations of $S_{\nu}\left(s^{\prime}, \theta, \varphi\right)$ and $I_{\nu}\left(s^{\prime}, \theta, \varphi\right)$ versus $s^{\prime}$ are only considered at spatial scales larger than $\delta$; these variations are negligible between $s^{\prime}-\delta$ and $s^{\prime}$. As the medium is considered as optically thick within the volume element 
of size $\delta$, we can consider that : i) the sum in Eq.19 is carried out with $s$ varying from $-\infty$ to $s^{\prime}$ (or $v=s^{\prime}-s$ varying from 0 to $\infty$ ), and write after some transformations

$$
S_{\nu}\left(s^{\prime}, \theta, \varphi\right)=I_{\nu}\left(s^{\prime}, \theta, \varphi\right)\left(\int_{0}^{\infty}\left[1-G_{e x t}(v)\right] \mathrm{d} v\right)^{-1}=B_{\nu} I_{\nu}\left(s^{\prime}, \theta, \varphi\right) .
$$

ii) in practice, $\frac{\mathrm{d} G_{\text {ext } \nu}}{\mathrm{d} s^{\prime}}, \frac{\mathrm{d} P_{s c \nu}}{\mathrm{d} s^{\prime}}$ and $\frac{\mathrm{d} P_{a \nu}}{\mathrm{d} s^{\prime}}$ are equal to zero for $s^{\prime}-s$ values larger than $\delta$, i.e. $G_{e x t \nu}, P_{s c \nu}$ and $P_{a \nu}$ have reached, in these conditions, their asymptotic values, 1 , $P_{s c \nu}(\infty)$ and $1-P_{s c \nu}(\infty)$, respectively. Eq.12 becomes, if we use Eqs.3, 10 and 15

$$
\begin{aligned}
\frac{\mathrm{d} I_{\nu}}{\mathrm{d} s^{\prime}}\left(s^{\prime}, \theta, \varphi\right)= & -S_{\nu}\left(s^{\prime}, \theta, \varphi\right)\left(\int_{s^{\prime}-\delta}^{s^{\prime}} \frac{\mathrm{d} G_{e x t \nu}}{\mathrm{d} s^{\prime}}\left(s^{\prime}-s\right) \mathrm{d} s\right) \\
& \left.+\Pi K_{\nu} n_{\nu}^{2} I_{\nu}^{\circ} T\left(s^{\prime}\right)\right] \\
& +\int_{4 \pi} S_{\nu}\left(s_{1}^{\prime}, \theta, \varphi\right)\left(\int_{s_{1}^{\prime}-\delta}^{s_{1}^{\prime}} \frac{\mathrm{d} P_{s c \nu}}{\mathrm{d} s_{1}^{\prime}}\left(s_{1}^{\prime}-s_{1}\right) \mathrm{d} s_{1}\right) \frac{p_{\nu}\left(\theta_{1}, \varphi_{1}, \theta, \varphi\right)}{4 \pi} \mathrm{d} \Omega_{1} .
\end{aligned}
$$

After simplification, we obtain, by introducing the asymptotic values of $G_{e x t \nu}$ and $P_{s c \nu}$ and Eqs. 8 and 20

$$
\begin{aligned}
\frac{\mathrm{d} I_{\nu}}{\mathrm{d} s^{\prime}}\left(s^{\prime}, \theta, \varphi\right)+B_{\nu} I_{\nu}\left(s^{\prime}, \theta, \varphi\right)= & \Pi K_{\nu} n_{\nu}^{2} I_{\nu}^{\circ}\left[T\left(s^{\prime}\right)\right] \\
& +\int_{4 \pi} \Sigma_{\nu} I_{\nu}\left(s_{1}, \theta_{1}, \varphi_{1}\right) \frac{p_{\nu}\left(\theta_{1}, \varphi_{1}, \theta, \varphi\right)}{4 \pi} \mathrm{d} \Omega_{1} .
\end{aligned}
$$

We find again, under the previous assumptions and for variations of $s^{\prime}$ at spatial scales larger than $\delta$, the classical formulation of the RTE, in which the extinction and scattering coefficients at equilibrium are given by Eqs.7 and 8. But Eq.22 is not valid for scales smaller than $\delta$, as far the medium does not follow Beer's laws. It can only be used in the asymptotic case of a locally thick medium, i.e., in practice, to introduce a radiative conductivity, as developed in the following section. These properties clearly appear in Fig.2. For an optical thickness larger than 2 to $3, G_{\text {ext }}$ is exponential and follows a Beer law, but in the semi-transparency thickness range $[0,2]$, this property is not true for a porosity $\Pi$ less than 0.6 .

Many experimenters have encountered the same type of behavior as in Fig.2. They often write that the extinction coefficient $\beta$, considered in a Beerian apprach, depends on the optical thickness of the medium, typically in the range $[0,2]$, and choose as $\beta$ determination the asymptotic value observed for an optical thickness greater than 2 . Though this value cannot be considered as a Beerian extinction coefficient and can be used neither in a classical RTE nor for calculating a radiative conductivity, it is nevertheless commonly done. 


\subsection{Radiative Fourier's law}

The study is here limited to the case of a porous medium with opaque and transparent phases at local scale, of porosity $\Pi$. The interface temperature field is called $T_{w}\left(x_{j}\right)$.

In so far as Eq.22 is a classical RTE, we can follow the approach of [20], here briefly summarized.

We introduce a non dimensional coordinate $x_{j}^{+}=x_{j} / \delta$ and a perturbation parameter $\mathrm{Kn}_{\nu}^{e x t}$, called extinction Knudsen number equal to $\left(B_{\nu} \delta\right)^{-1}$, by analogy with the Boltzmann's equation formalism. $\mathrm{Kn}_{\nu}^{e x t}$ is small in front of 1 , in so far as the medium is optically thick, and we assume that the solution of Eq.22 is written

$$
I_{\nu}\left(x_{j}^{+}, \theta, \varphi\right)=I_{\nu}^{(0)}\left(x_{j}^{+}, \theta, \varphi\right)+I_{\nu}^{(1)}\left(x_{j}^{+}, \theta, \varphi\right)
$$

where $I_{\nu}^{(0)}$ is the RTE's solution at the zero order of perturbation versus $\mathrm{Kn}^{\text {ext }}$, equal to $\Pi n_{\nu}^{2} I_{\nu}^{\circ}\left[T_{w}\left(x_{j}^{+}\right)\right]$. The contribution to the radiative flux, given by Eq.16, of the zero order solution $I_{\nu}^{(0)}$ is null in so far as it corresponds to LTE conditions of the radiation field, which are similar to the LTE conditions of the material system, commonly used in heat transfer. From a physical point of view, when the coupling of the considered phase within a volume element, of size $\delta$, with the surrounding volume elements are neglected, this phase can be characterized by an equilibrium intensity, at temperature $T_{w}\left(x_{j}\right)$.

In these conditions, the solution of Eq.22 at the first order versus $\mathrm{Kn}_{\nu}^{e x t}$ is obtained using the zero order intensity in the first term of the first member of this equation and by only keeping terms proportional to $\mathrm{Kn}_{\nu}^{e x t}$, i.e.

$$
\begin{aligned}
I_{\nu}^{(1)}\left(x_{j}^{+}\right)= & -\mathrm{Kn}_{\nu}^{e x t} u_{i} \Pi n_{\nu}^{2} \frac{\partial I_{\nu}^{\circ}}{\partial x_{i}^{+}}\left[T_{w}\left(x_{j}^{+}\right)\right] \\
& +\frac{1}{4 \pi B_{\nu}} \int_{0}^{\pi} \int_{0}^{2 \pi} \Sigma_{\nu} I_{\nu}^{(1)}\left(x_{j}^{+}, \theta_{1}, \varphi_{1}\right) p_{\nu}\left(\theta_{1}, \varphi_{1}, \theta, \varphi\right) \sin \theta_{1} \mathrm{~d} \theta_{1} \mathrm{~d} \varphi_{1} .
\end{aligned}
$$

Equation 24 shows that the perturbation first order implicit solution $I_{\nu}^{(1)}$, which can be obtained by iteration, is proportional to $-u_{i} \Pi \frac{\partial I_{\nu}^{\circ}}{\partial x_{i}^{+}}\left[T_{w}\left(x_{j}^{+}\right)\right]$and consequently to $-u_{i} \frac{\partial T}{\partial x_{i}}\left(x_{j}\right)$. Finally, by introducing the solution $I_{\nu}^{(1)}$ of Eq.24 in Eq.16, we obtain the radiative Fourier law

$$
q_{i}^{R}\left(x_{j}\right)=-k_{i l}^{R} \frac{\partial T}{\partial x_{l}}\left(x_{j}\right),
$$

in which appears the conductivity tensor $k_{i l}^{R}$. Due to the system symmetries, this tensor is generally diagonal. For statistically isotropic porous media, it is simply a scalar as in the case of the phonon conductivity theory.

Radiation is generally coupled to other heat transfer modes within the porous medium. 
We only consider here the coupling terms in the case, considered in the next section, of a medium with opaque and transparent phases at local scale; Eq.24 is then applied to the propagation phase, i.e. the transparent fluid phase of the real medium. Consequently, an equivalent radiative power per unit volume is, in principle, introduced within this phase, given by Eq.17.

In fact, as the radiative transfer occurs at the opaque interfaces, characterized by the temperature field $T_{w}\left(x_{i}\right)$, the radiative flux per unit interfacial area within the considered volume element is given by Eq.18, i.e. in the present conditions by

$$
\varphi^{R}\left(x_{j}\right)=\frac{1}{A} \frac{\partial}{\partial x_{i}}\left[k_{i l} \frac{\partial T_{w}}{\partial x_{i}}\right]\left(x_{j}\right)
$$

where $A$ is the specific fluid area per unit volume of the porous medium. It is worth noticing that this model corresponds to a porous medium in non equilibrium, characterized by the temperatures associated with the solid phase $T_{S}\left(x_{i}\right)$ and the transparent fluid phase $T_{F}\left(x_{i}\right)$, and also by $T_{w}\left(x_{i}\right)$. On the other hand, if the whole volume element of the porous medium is assumed to be at equilibrium at temperature $T$, the simplest way is to consider an effective radiative power per unit volume, i.e.

$$
P^{R}\left(x_{j}\right)=\frac{\partial}{\partial x_{i}}\left[k_{i l} \frac{\partial T}{\partial x_{l}}\right]\left(x_{j}\right) .
$$

In the most common case, the phase function $p_{\nu}\left(\mu_{s c}\right)$ of the porous medium, assumed statistically isotropic, only depends on the scattering angle cosine $\mu_{s c}$. In these conditions, there is an analytical expression of the radiative diffusion flux, established in Appendix $\mathrm{B}$, for an optically thick medium at local scale. The associated isotropic conductivity is then given, for a porous medium in non equilibrium conditions, by

$$
k^{R}\left(T_{w}\right)=\frac{4 \pi \Pi}{3} \int_{0}^{\infty} \frac{n_{\nu}^{2}}{K_{\nu}+\Sigma_{\nu}\left(1-g_{\nu}\right)} \frac{\mathrm{d} I_{\nu}^{\circ}}{\mathrm{d} T_{w}}\left(T_{w}\right) \mathrm{d} \nu,
$$

in which $g_{\nu}$ is the asymmetry factor of scattering, classically defined by

$$
g_{\nu}=\frac{1}{2} \int_{-1}^{1} p_{\nu}\left(\mu_{s c}\right) \mu_{s c} \mathrm{~d} \mu_{s c}
$$

It is worth noticing that, to our knowledge, there is no accurate validity criterion of the use of radiative Fourier's law, in particular for a strongly scattering medium, case of any porous medium. This point is a key current research field. 


\section{Examples of application}

\subsection{Overlapping Transparent Spheres within an opaque solid phase}

Statistically isotropic foams, for instance mullite in most spectral bands, can be modeled by a set of DOTS. These overlapping spheres are characterized by dispersed radii and random locations of their centers. Tancrez and Taine [14] have shown, using the RDFI approach, that the radiative transfer between the opaque phase interfaces can be modeled using an effective Beerian semi transparent medium within the real transparent phase only when the medium porosity is greater than 0.6. $G_{\text {ext }}$ plotted in Fig.2, and consequently $\beta$, do not depend on the frequency $\nu$, which is a classical result. It has also been established that the phase function $p_{\nu}\left(\mu_{s c}\right)$ only depends on the scattering angle cosine $\mu_{s c}$. On the other hand, it is obvious that the radiative conduction model is more valuable as the porosity is small. The purpose of this section is to develop the radiative conductivity model for all the porosity values from results of Sec.4.2.

The cumulative extinction distribution functions $G_{e x t}$ and phase functions $p_{\nu}\left(\mu_{s c}\right)$ obtained for DOTS configurations versus the medium porosity $\Pi$ in [14] are the starting point of this study. The extinction coefficient at equilibrium $B$ defined by Eq.7 is also independent of the frequency and normalized by the asymptotic value $\beta_{O T}$ associated with an optically thin medium, equal to $A /(4 \Pi)$, as in [14], i.e.

$$
B^{+}=\frac{B}{\beta_{O T}}=\frac{4 \Pi B}{A} \quad ; \quad \beta^{+}=\frac{\beta}{\beta_{O T}} .
$$

[Figure 5 about here.]

$B^{+}$is obtained from Eq.7, i.e. by the calculation of the area under the curve $1-G_{\text {ext }}$, with a relative accuracy better than $10^{-4}$. The results are shown in Fig.5 and compared to the previous results of [14], related to $\beta^{+}$and obtained from the RDFI approach, in which a Beer law is assumed. $\beta^{+}$has been extracted from a least square fit of $1-G_{\text {ext }}$ by an exponential function in the range $[0,3]$ of $\beta_{O T} s$, i.e. approximately in the range $[0,4.5]$ of $\beta$ s. The main interest of this RDFI approach is to introduce an accurate quantitative criterion of the validity of Beer's laws, which is the standard deviation $\varepsilon_{\beta^{+}}$on $\beta^{+} . \varepsilon_{\beta^{+}}$is also plotted in Fig.5. It is worth noticing that the results related to $B^{+}$and $\beta^{+}$converge when the porosity increases, i.e. when $1-G_{e x t}$ becomes close to an exponential. The two determination techniques are then equivalent. In the other hand, $\beta^{+}$and $B^{+}$have no physical meaning for intermediate or low porosity values, as long as $1-G_{\text {ext }}$ is far from being exponential in the variation range $[0,2]$ of $\beta s$. But the determination of $B^{+}$is useful for calculating the radiative conductivity of locally optically thick media, especially in the case of a low porosity. 
Let us first consider a diffuse reflection law for the solid phase, defined by a hemispherical absorptivity $\alpha_{\nu}^{h}$. In this particular case, characterized by the phase function given in [14], the asymmetry factor of scattering is a scalar, independent of $\alpha_{\nu}^{h}$, and consequently of $\nu$, equal to $g^{\text {diff }}=-0.1827$.

The isotropic conductivity is then deduced from Eqs. 28 and 30, i.e.

$$
k^{R \operatorname{diff}}\left(T_{w}, \Pi, A\right)=\frac{\Pi^{2}}{A} \frac{16 \pi}{3} \frac{1}{B^{+}(\Pi)} \int_{0}^{\infty} \frac{n_{\nu}^{2}}{\alpha_{\nu}^{h}+\left(1-\alpha_{\nu}^{h}\right)\left(1-g^{\text {diff }}\right)} \frac{\mathrm{d} I_{\nu}^{\circ}}{\mathrm{d} T_{w}}\left(T_{w}\right) \mathrm{d} \nu,
$$

and under the assumption of a gray solid phase characterized by $\alpha^{h}$

$$
k^{R \operatorname{diff}}\left(T_{w}, \Pi, A\right)=\frac{\Pi^{2}}{A} \frac{64 \pi}{3} \frac{1}{B^{+}(\Pi)} \frac{n^{2} \sigma T_{w}^{3}}{\alpha^{h}+\left(1-\alpha^{h}\right)\left(1-g^{\operatorname{diff} f}\right)} .
$$

For DOTS, $A$ is linked with the distribution of the radii $R$ by [25]

$$
A=\frac{3<R^{2}>}{<R^{3}>}(1-\Pi) \ln \left(\frac{1}{1-\Pi}\right)
$$

where $<R^{2}>$ and $<R^{3}>$ are the average values of $R^{2}$ and $R^{3}$.

Let us consider now a specular reflection law, simply modeled by

$$
\alpha_{\nu}^{\text {spec }}=1-\rho_{\nu}^{\text {spec }}=\frac{3}{2} \alpha_{\nu}^{h} \mu_{i}
$$

where $\mu_{i}$ is the incidence angle cosine at local scale. The scattering parameter $g_{\nu}^{\text {spec }}$ associated with the corresponding phase function given in [14] now depends on $\alpha_{\nu}^{h}$; it varies in the range $[-0.078,0.15]$ and is given by

$$
g_{\nu}^{\text {spec }}=0.8913 \alpha_{\nu}^{h^{3}}-0.4110 \alpha_{\nu}^{h^{2}}+0.1688 \alpha_{\nu}^{h}-0.07772
$$

result obtained by a least square fit with a relative standard deviation of 0.013 . It is worth noticing that the specular phase function is, in practice, close to an isotropic phase function, i.e. the effect of the scattering source term is weak. A similar result has been obtained for a diffuse reflection law.

From the asymptotic value $P_{a \nu}(\infty)$ of the cumulative absorption probability, we have numerically determined, paralely to $G_{\text {ext }}$ in the Monte Carlo approach, a normalized absorption coefficient at equilibrium $K^{+}$equal to $K_{\nu}(\Pi) /\left[B(\Pi) \alpha_{\nu}^{h}\right]$ and independent of $\nu$. The effective conductivity associated with the specular reflection law is then given by Eqs. 31-32, where the quantity $\alpha_{\nu}^{h} K^{+}(\Pi)+\left[1-\alpha_{\nu}^{h} K^{+}(\Pi)\right]\left(1-g_{\nu}^{\text {spec }}\right)$ is substituted for $\alpha_{\nu}^{h}+\left(1-\alpha_{\nu}^{h}\right)\left(1-g^{\operatorname{diff}}\right)$.

The ratio $\left[\alpha_{\nu}^{h} K^{+}(\Pi)+\left[1-\alpha_{\nu}^{h} K^{+}(\Pi)\right]\left(1-g_{\nu}^{\text {spec }}\right)\right] /\left[\alpha_{\nu}^{h}+\left(1-\alpha_{\nu}^{h}\right)\left(1-g^{\text {diff }}\right)\right]$ has been 
computed versus $\Pi$ and $\alpha^{h}$ varying in the ranges [0.1,0.82] and [0,2/3] respectively. It is worth noticing that this ratio, equal to 1.098 with a discrepancy less than 0.003 , is in practice independent of both $\Pi$ and $\alpha^{h}$. Consequently, conductivity results associated with a specular reflection law can be deduced from results associated with a diffuse reflection law using this simple multiplication factor.

\subsection{Bundle of rods}

The radiative properties of bundles of opaque rods, in triangular or square configuration (see Figs.1(a), 1(b) and 1(c)), have been studied by Bellet et al. [20], by considering effective anisotropic extinction, absorption and scattering coefficients, i.e. an equivalent Beerian semi transparent medium.

But, as shown in Fig.3, this approach is a poor approximation; $G_{\text {ext }}\left(s^{\prime}-s, \theta, \varphi\right)$ is far from being exponential, in particular in a cross section of a rod bundle characterized by $\theta=\pi / 2$, for azimuthal directions $\varphi$ corresponding to alleys parallel to the lines joining the centers of the rod cross sections. $\varphi=0^{\circ}$ corresponds to a center line; due to the system symmetry, $\varphi$ belongs to the range $\left[0^{\circ}, 30^{\circ}\right]$ or $\left[0^{\circ}, 45^{\circ}\right]$ for a triangular or a square configuration respectively. More precisely, $\ln \left[1-G_{e x t}\left(s^{\prime}-s, \pi / 2, \varphi\right)\right]$ is plotted in Fig. 3 versus $\beta_{O T}\left(s^{\prime}-s\right)$, where $\beta_{O T}$ is the Beerian extinction coefficient at the limit of an optically thin system, equal to $A / \pi$, as introduced in [20]. Discrepancies with exponential functions can be extremely important in Fig.3. The aim of this section is to apply to this system, considered as optically thick at local scale, the diffusion approximation based on the GRTE and the derived rigorous expressions of $B, K_{\nu}$ and $\Sigma_{\nu}$.

We only consider, for both square and triangular configurations of opaque rod bundles, a diffuse reflection law characterized by the hemispherical absorptivity $\alpha_{\nu}^{h}$. The study is only carried out in a cross section plane $(\theta=\pi / 2)$, as far it has been established that, for this infinite system, elements of a conductivity tensor cannot be defined for the rod axis direction [20]. As $B(\pi / 2, \varphi), K_{\nu}(\pi / 2, \varphi)$ and $\Sigma_{\nu}(\pi / 2, \varphi)$, equal to $\alpha_{\nu}^{h} B(\pi / 2, \varphi)$ and $\left(1-\alpha_{\nu}^{h}\right) B(\pi / 2, \varphi)$ respectively, strongly depend on $\varphi$, an effective real index $n_{\nu}(\pi / 2, \varphi)$ depending on $\varphi$ is calculated by the method detailed in Appendix A. For system symmetry reasons, the emission term $K_{\nu}(\pi / 2, \varphi) n_{\nu}^{2}(\pi / 2, \varphi) I_{\nu}^{\circ}\left(T_{w}\right)$ is isotropic [20]. Consequently Eq.24 and the results of Sec.4.2 can be applied.

The main difficulty of this part is to determine with accuracy the integral (area) between 0 and $\infty$ of the function $1-G_{\text {ext }}\left(s^{\prime}-s, \pi / 2, \varphi\right)$ that defines $B(\pi / 2, \varphi)$ in Eq.7. A critical optical thickness $B s_{\text {crit }}$ at $95 \%$ (resp. 99\%), shown in Fig.6a, is associated to the distance $s_{\text {crit }}$ at which the calculation gives a value of $95 \%$ (resp. 99\%) of the area between 0 and $\infty$. Values of this optical thickness as large as 80 are required in some particular directions, which correspond to alleys between rods, due to the low convergence of $1-G_{\text {ext }}$. 


\section{[Figure 6 about here.]}

The strong variations of $B$ versus $\varphi$ in a bundle cross section are illustrated in Fig.6b. After the determination of $B, K_{\nu}$ and $\Sigma_{\nu}$, the procedure detailed in [20] has been followed to accurately compute the radial conductivity of the rod bundles, isotropic in a cross section due to the system symmetry. In fact, we have introduced and tabulated the normalized quantity $k^{R+}\left(\Pi, \alpha_{\nu}^{h}\right)$ defined in this reference, such as

$$
k^{R}\left(T_{w}, \Pi, A, \alpha_{\nu}^{h}\right)=\frac{\pi \Pi^{2}}{A} \int_{0}^{\infty} \frac{\mathrm{d} I_{\nu}^{\circ}}{\mathrm{d} T_{w}}\left(T_{w}\right) k^{R+}\left(\Pi, \alpha_{\nu}^{h}\right) \mathrm{d} \nu
$$

The results are summarized in Table.1 versus the porosity $\Pi$ and $\alpha_{\nu}^{h}$ for the triangular and the square configurations. It is worth noticing that they often strongly differ from those of [20]: this is due to the very large discrepancies of this type of system with an effective Beerian semi transparent medium.

[Table 1 about here.]

\section{Conclusion}

This paper deals with homogenization of the radiation field in a porous medium, even if the homogenized phases do not follow Beer's laws related to extinction, absorption and scattering. This approach is limited to media characterized by typical pore sizes larger than the useful radiation wavelengths; diffraction and coherence effects are neglected and the geometrical optics laws are valid. The study is also limited to statistically isotropic and statistically uniform porous media. The developed approach is general, but has been here mainly applied to the case of porous media with an opaque phase and a transparent one. The main results are:

i) The radiative properties of any homogenized phase can be characterized by three radiative statistical functions : extinction cumulative distribution function, scattering cumulative probability and general scattering phase function; an effective refractive index has to be introduced for statistically anisotropic porous media.

ii) The spatial resolution of this radiative homogenization is only limited by the accuracy on the determination of the distances from any point of a phase to any other point or interface of this phase, in a Monte Carlo method of calculation of the previous radiative statistical functions. In most cases, this limitation is linked to the spatial resolution of a $\mathrm{X}$ or $\gamma$ tomography applied to the considered porous medium. The determination of the radiative statistical functions also requires to know the medium radiative properties at a local scale, lower than the scales accounted for by the tomography techniques.

iii) The intensity field within any homogenized phase is expressed from a Generalized Radiative Transfer Equation (GRTE), which depends on the three previous statistical 
functions and on the emission terms, functions of the homogenized temperature field for a material system in Local Thermal Equilibrium (LTE) conditions. This temperature field, associated with the material system, has to be homogenized from the classical homogenization techniques used in porous media, generally characterized by greater spatial resolution scales. The two independent homogenization techniques, applied to the radiation field and the material system, are compatible.

iv) An important established result is that, for a large optical thickness, any GRTE degenerates in a classical Radiative Transfer Equation (RTE), even for non Beerian homogenized phases of a porous medium. This asymptotic RTE is characterized by generalized extinction and scattering coefficients at equilibrium, expressed versus the radiative statistical functions.

v) In the case of a homogenized phase optically thick at a scale such as it can be practically considered isothermal, the radiative transfer can be modeled by a radiative Fourier law, based on a radiative conductivity tensor. This tensor is directly deduced, by a perturbation technique, similar to the Chapmann Enskog model, from the generalized extinction and scattering coefficients at equilibrium. Two application examples have been given; they are related to a set of Dispersed Opaque Transparent Spheres (DOTS) within an opaque phase and to rod bundles.

vi) Precise validity conditions of the radiative Fourier law have to be defined for practical applications versus the temperature field, the absorption and extinction coefficients, possibly generalized at equilibrium, and the phase function. It is a current key research field.

\section{Appendix A. Determination of $n_{\nu}(\theta, \varphi)$}

We consider a statistically anisotropic porous medium, characterized by $B_{\nu}(\theta, \varphi)$, $K_{\nu}(\theta, \varphi), \Sigma_{\nu}(\theta, \varphi)$ and $n_{\nu}(\theta, \varphi)$, all depending on the current direction $(\theta, \varphi) \cdot n_{\nu}(\theta, \varphi)$ can be determined by expressing the equality between the extinction term due to scattering and the scattering source term in equilibrium conditions of an optically thick medium at temperature T. Using Eqs.3, 6, 10 and 15, we obtain

$$
\begin{aligned}
& S_{\nu}^{\circ}(\theta, \varphi) \\
= & B_{\nu}(\theta, \varphi) n_{\nu}^{2}(\theta, \varphi) I_{\nu}^{\circ}(T) \\
= & K_{\nu}(\theta, \varphi) n_{\nu}^{2}(\theta, \varphi) I_{\nu}^{\circ}(T)+\int_{4 \pi} \int_{s_{10}}^{s_{1}^{\prime}} \frac{\mathrm{d}\left[P_{s c \nu}\left(s_{1}^{\prime}-s_{1}, \theta_{1}, \varphi_{1}\right)\right]}{\mathrm{d} s_{1}^{\prime}} \frac{p_{\nu}\left(\theta_{1}, \varphi_{1}, \theta, \varphi\right)}{4 \pi} S_{\nu}^{\circ}(\theta, \varphi) \mathrm{d} s_{1} \mathrm{~d} \Omega_{1} .
\end{aligned}
$$


After the spatial summation over the considered optically thick medium at equilibrium, Eq.A.1 becomes

$\Sigma_{\nu}(\theta, \varphi) n_{\nu}^{2}(\theta, \varphi) I_{\nu}^{\circ}(T)=\int_{4 \pi} \frac{p_{\nu}\left(\theta_{1}, \varphi_{1}, \theta, \varphi\right)}{4 \pi} P_{s c \nu}\left(\infty, \theta_{1}, \varphi_{1}\right) B_{\nu}\left(\theta_{1}, \varphi_{1}\right) n_{\nu}^{2}\left(\theta_{1}, \varphi_{1}\right) I_{\nu}^{\circ}(T) \mathrm{d} \Omega_{1}$

and finally, using Eq.8

$$
\Sigma_{\nu}(\theta, \varphi) n_{\nu}^{2}(\theta, \varphi)=\int_{4 \pi} \frac{p_{\nu}\left(\theta_{1}, \varphi_{1}, \theta, \varphi\right)}{4 \pi} \Sigma_{\nu}\left(\theta_{1}, \varphi_{1}\right) n_{\nu}^{2}\left(\theta_{1}, \varphi_{1}\right) \mathrm{d} \Omega_{1}
$$

This last implicit equation allows us to determine $n_{\nu}^{2}(\theta, \varphi)$. It is a generalization of the equation used for a Beerian semi transparent medium (see [20]).

\section{Appendix B. Radiative conductivity of a spatially isotropic medium}

The aim of this Appendix is to express the radiative conductivity of a non Beerian medium characterized by isotropic extinction, absorption and scattering coefficients at equilibrium $B_{\nu}, K_{\nu}$ and $\Sigma_{\nu}$, an isotropic optical index $n_{\nu}$ and also characterized by a phase function only depending on the scattering cosine angle $\mu_{s c}$. This approach is obviously also valid for an optically thick volume element of a Beerian medium characterized by $\beta_{\nu}, \kappa_{\nu}$ and $\sigma_{\nu}$ and $n_{\nu}$.

We consider a volume element, of typical size $\delta$, optically thick and isothermal at the zero perturbation order, under the conditions defined in Sec.4.2. The first order perturbation solution, defined by Eq.24, becomes

$$
\begin{aligned}
I_{\nu}^{(1)}\left(x_{j}, \theta, \varphi\right)= & -\frac{n_{\nu}^{2}}{B_{\nu}} u_{i} \frac{\partial I_{\nu}^{\circ}}{\partial x_{i}}\left[T_{w}\left(x_{j}\right)\right] \\
& +\frac{\Sigma_{\nu}}{4 \pi B_{\nu}} \int_{0}^{\pi} \int_{0}^{2 \pi} I_{\nu}^{(1)}\left(x_{j}, \theta_{1}, \varphi_{1}\right) p_{\nu}\left(u_{1 l} u_{l}\right) \sin \left(\theta_{1}\right) \mathrm{d} \theta_{1} \mathrm{~d} \varphi_{1} .
\end{aligned}
$$

In the last sum of this equation, $u_{1 l} u_{l}$ is the cosine of the scattering angle between the directions $\left(\theta_{1}, \varphi_{1}\right)$ and $(\theta, \varphi)$. The spectral radiative flux defined by Eq.16 can then be written

$$
\begin{aligned}
\mathrm{d} q_{k \nu}^{R}\left(x_{j}\right)= & \int_{0}^{\pi} \int_{0}^{2 \pi} I_{\nu}^{(1)}\left(x_{j}, \theta, \varphi\right) u_{k} \sin \theta \mathrm{d} \theta \mathrm{d} \varphi \mathrm{d} \nu \\
= & -\frac{n_{\nu}^{2}}{B_{\nu}} \int_{0}^{\pi} \int_{0}^{2 \pi} u_{i} \frac{\partial I_{\nu}^{\circ}}{\partial x_{i}}\left[T_{w}\left(x_{j}\right)\right] u_{k} \sin \theta \mathrm{d} \theta \mathrm{d} \varphi \mathrm{d} \nu \\
& +\frac{\Sigma_{\nu}}{4 \pi B_{\nu}} \int_{0}^{\pi} \int_{0}^{2 \pi}\left[\int_{0}^{\pi} \int_{0}^{2 \pi} I_{\nu}^{(1)}\left(x_{j}, \theta_{1}, \varphi_{1}\right) p_{\nu}\left(u_{1 l} u_{l}\right) \sin \theta_{1} \mathrm{~d} \theta_{1} \mathrm{~d} \varphi_{1}\right] u_{k} \sin \theta \mathrm{d} \theta \mathrm{d} \varphi \mathrm{d} \nu
\end{aligned}
$$


The last term of Eq.B.2 can be written by permuting the sums

$\mathcal{J}_{\nu}=\frac{\Sigma_{\nu}}{4 \pi B_{\nu}} \int_{0}^{\pi} \int_{0}^{2 \pi} I_{\nu}^{(1)}\left(x_{j}, \theta_{1}, \varphi_{1}\right) \quad\left[\int_{0}^{\pi} \int_{0}^{2 \pi} u_{k} p_{\nu}\left(u_{1 l} u_{l}\right) \sin \theta \mathrm{d} \theta \mathrm{d} \varphi\right] \sin \theta_{1} \mathrm{~d} \theta_{1} \mathrm{~d} \varphi_{1} \mathrm{~d} \nu(\mathrm{B} .4)$

For symmetry reasons, we can write

$$
\int_{0}^{\pi} \int_{0}^{2 \pi} u_{k} p_{\nu}\left(u_{1 l} u_{l}\right) \sin \theta \mathrm{d} \theta \mathrm{d} \varphi=4 \pi g_{\nu} u_{1 k},
$$

where $g_{\nu}$ is the scattering parameter defined by Eq.29. Consequently, $\mathcal{J}$ becomes

$$
\mathcal{J}_{\nu}=\frac{\Sigma_{\nu} g_{\nu}}{B_{\nu}} \mathrm{d} q_{k \nu}^{R}\left(x_{j}\right)
$$

Finally from Eqs.B.2 -B.5 we obtain, by accounting for the orthogonality rules of the $u_{i}$

$$
q_{k}^{R}\left(x_{j}\right)=-\frac{4 \pi}{3} \int_{0}^{\infty} \frac{n_{\nu}^{2}}{B_{\nu}-\Sigma_{\nu} g_{\nu}} \frac{\partial I_{\nu}^{\circ}}{\partial x_{k}}\left[T_{w}\left(x_{j}\right)\right] \mathrm{d} \nu,
$$

which leads to the radiative Fourier law

$$
q_{k}^{R}\left(x_{j}\right)=-k^{R} \frac{\partial T}{\partial x_{k}} \mathrm{~d} \nu
$$

by introducing the radiative conductivity

$$
k^{R}=\frac{4 \pi}{3} \int_{0}^{\infty} \frac{n_{\nu}^{2}}{B_{\nu}-\Sigma_{\nu} g_{\nu}} \frac{\partial I_{\nu}^{\circ}}{\partial T_{w}}\left[T_{w}\left(x_{j}\right)\right] \mathrm{d} \nu .
$$

\section{References}

[1] R. Coquard, D. Baillis, Radiative characteristics of beds of spheres containing an absorbing and scattering medium, Journal of Thermophysics and Heat Transfer 19 (2005) 226-234.

[2] R. Coquard, D. Baillis, Radiative properties of dense fibrous medium containing fibers in the geometric limit, Journal of Thermophysics and Heat Transfer (2006) $1022-1030$.

[3] T. Hendricks, J. Howell, Inverse radiative analysis to determine spectral radiative properties using the discrete ordinates method, International Journal of Heat and Mass Transfer 2 (1994) 75-80.

[4] T. J. Hendricks, J. R. Howell, Absorption/scattering coefficients and scattering 
phase function in reticulated porous ceramics, ASME J.of Heat Transfer. 118 (1996) $79-87$.

[5] T. Hendricks, J. Howell, New radiative analysis approach for reticulated porous ceramics using discrete ordinates method, ASME J.of Heat Transfer 4 (1996) 911917.

[6] D. Baillis, J. Sacadura, Identification of spectral radiative properties of polyurethane foam from hemispherical and bi-directional transmittance and reflectance measurement, Journal of Quantitative Spectroscopy and Radiative Transfer 73 (2002) 297306.

[7] B. P. Singh, M. Kaviany, Modelling radiative heat transfer in packed beds, International Journal of Heat and Mass Transfer 6 (1992) 1397-1405.

[8] M. Loretz, R. Coquard, D. Baillis, D. Maire, Metallic foams : radiative properties; comparison between different models, Journal of Quantitative Spectroscopy and Radiative Transfer 109 (2008) 16-27.

[9] D. Baillis, J. F. Sacadura, Thermal radiation properties of dispersed media: theoretical prediction and experimental characterization, Journal of Quantitative Spectroscopy and Radiative Transfer 67 (2000) 327-363.

[10] J. Consalvi, B. Porterie, J. Loraud, A formal averaging procedure for radiation heat transfer in particulate media, International Journal Heat and Mass Transfer 45 (2002) 2755-276!

[11] S. Whitaker, The method of volume averaging, Kluwer Academics Publishers, 1999.

[12] M. Quintard, S. Whitaker, One-and two equationmodels in two phase systems, volume 23 of Advances in Heat Transfer, pp. 369-464.

[13] W. Lipinski, J. Petrasch, S. Haussener, Application of the spatial averaging theorem to radiative heat transfer in two-phase media, Journal of Quantitative Spectroscopy and Radiative Transfer 11 (2010) 253-258.

[14] M. Tancrez, J. Taine, Direct identification of absorption and scattering coefficients and phase function of a porous medium by a Monte Carlo technique, International Journal of Heat and Mass Transfer 47 (2004) 373-383.

[15] B. Zeghondy, E. Iacona, J. Taine, Determination of the anisotropic radiative properties of a porous material by radiative distribution function identification (RDFI), International Journal of Heat and Mass Transfer 49 (2006) 2810-2819. 
[16] B. Zeghondy, E. Iacona, J. Taine, Experimental validation of RDFI method predictions of statistically anisotropic porous medium radiative properties, International Journal of Heat and Mass Transfer 49 (2006) 3701-3707.

[17] J. Petrasch, P. Wyss, A. Steinfeld, Tomography-based Monte Carlo determination of radiative properties of reticulated porous ceramics, Journal of Quantitative Spectroscopy and Radiative Transfer 105 (2007) 180-197.

[18] S. Haussener, P. Coray, W. Lipinski, P. Wyss, A. Steinfeld, Tomography-based heat and mass transfer characterization of reticulate porous ceramics for hightemperature processing, J. of Heat Transfer 131 (2009) 1-9.

[19] S. Haussener, W. Lipinski, P. Wyss, A. Steinfeld, Tomography-based analysis of radiative transfer in reacting packed beds undergoing a solid-gas thermochemical transformation, J. of Heat Transfer in press (2009).

[20] F. Bellet, E. Chalopin, F. Fichot, E. Iacona, J. Taine, RDFI determination of anisotropic and scattering dependent radiative conductivity tensors in porous media: Application to rod bundles, International Journal of Heat and Mass Transfer 52 (2009) 1544-1551.

[21] A. Gusarov, Homogenization of radiation transferin two-phase media with irregular phase boundaries, Phys.Rev. B 77 (2008) 144201.

[22] H. Scheurer, Data Book PHEBUS FPT1, Issue J-01.09.95 IS.92.49, CEA, Cadarache, France, 1995.

[23] J. Ferziger, N. Kaper, Mathematical Theory of Transport Processes in Gases, Elsevier, U.S.A., 1972.

[24] S. Chapman, T. Cooling, The Mathematical Theory of Non-uniform Gases, Cambridge University Press, 1962.

[25] S. Torquato, B. Lu, Chord-length distribution for two phase random media,, Phys. Rev. E 4 (1993) 47. 


\section{List of Figures}

.1 Shooting zones for a) triangular and b) square rod bundle configurations, delimited by bold lines; c) system of coordinates. . . . . . . . . . . . . 28

.2 Extinction cumulative distribution for DOTS model; $\Pi$ from left to right: $0.18,0.26,0.37,0.48,0.56,0.65,0.72,0.78,0.82$; data of $[14] \ldots 29$

.3 Extinction cumulative distribution functions $G_{e x t}(s, \pi / 2, \varphi)$ of a rod bundle for different values of $\varphi\left(1.5^{\circ} \square, 7.5^{\circ}+, 13.5^{\circ} \circ, 22.5^{\circ} \times, 28.5^{\circ} \triangle\right)$; triangular pattern; a) $\Pi=0.1500$, b) $\Pi=0.5130$ and c) $\Pi=0.7500$. Extinction cumulative distribution function $G_{\text {ext }}\left(s^{\prime}-s, \pi / 2\right)$ for all values of $\varphi$ (thick line). Extinction cumulative distribution function $G_{\text {ext }}\left(s^{\prime}-s, \pi / 2\right)$ of the Beerian semi transparent medium such as $\beta(\pi / 2)=B(\pi / 2)$ (thin line . . . . . . . . . . . . . . . . . . . 30

.4 Scattering by a volume element: a)extinction by the volume element ; b) source term . . . . . . . . . . . . . . . . 31

.5 Convergence of calculated $B^{+}(+)$and $\beta^{+}(\square)$ issued from [14]; relative standard deviation $\varepsilon_{\beta^{+}}$associated with $\beta^{+} \ldots \ldots . \ldots . \ldots . \ldots 32$

.6 a) Critical optical thickness $B s_{c r i t}(\pi / 2, \varphi)$ associated with the distance $s_{\text {crit }}$ such as the area under $1-G_{e x t}$ is $95 \%$ of its value at infinity $(\triangle$ triangular, $\square$ square pattern) and at $99 \%$ ( $\boldsymbol{\Delta}$ triangular, $\square$ square pattern); $\Pi=0.5130$. b) Normalized generalized extinction coefficient at equilibrium $B^{+}(\pi / 2, \varphi)=\pi B^{+}(\pi / 2, \varphi) A_{F}$; triangular pattern; $\Pi=0.3000$ : + , $\Pi=0.5130: \Delta, \Pi=0.9000: \times \ldots \ldots \ldots \ldots \ldots$ 

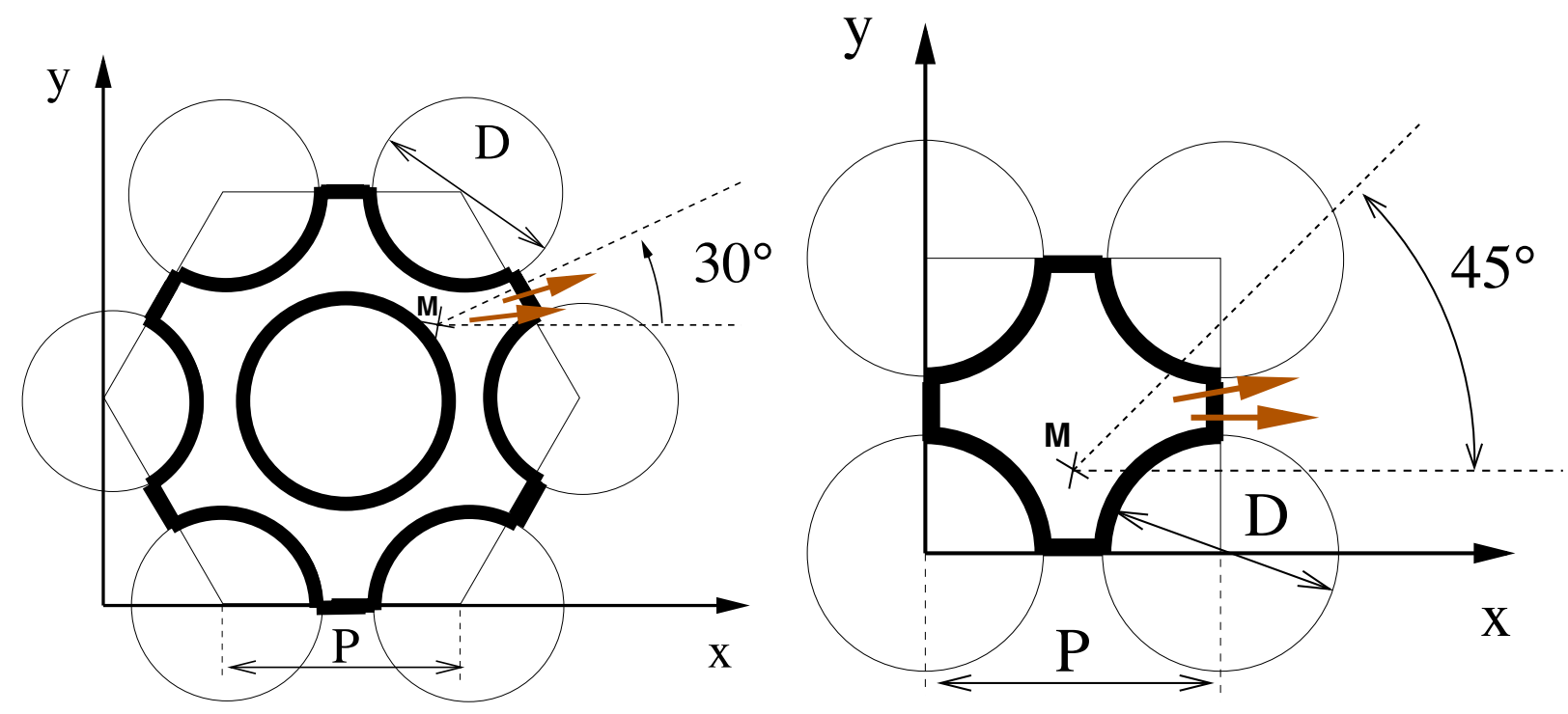

(a)

(b)

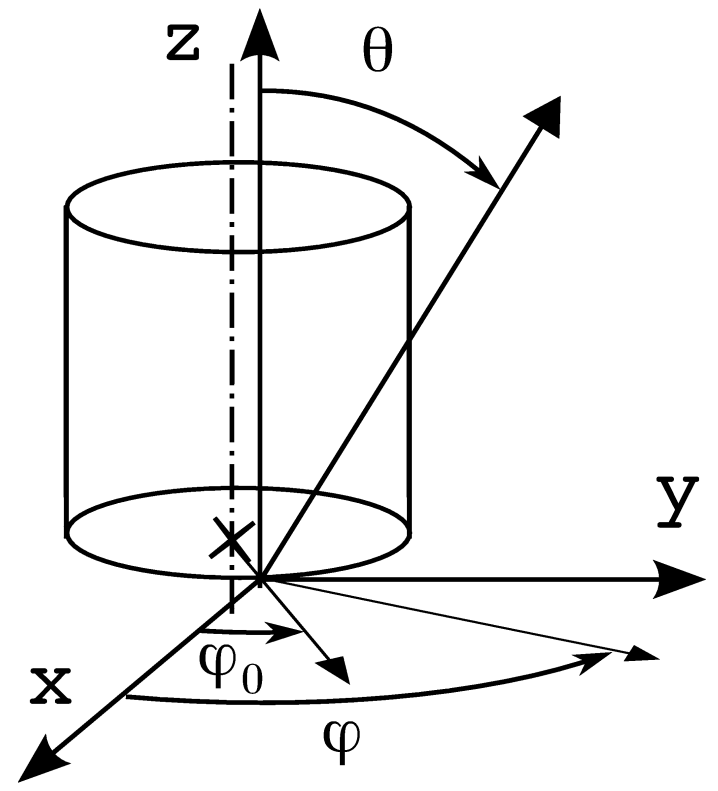

(c) 


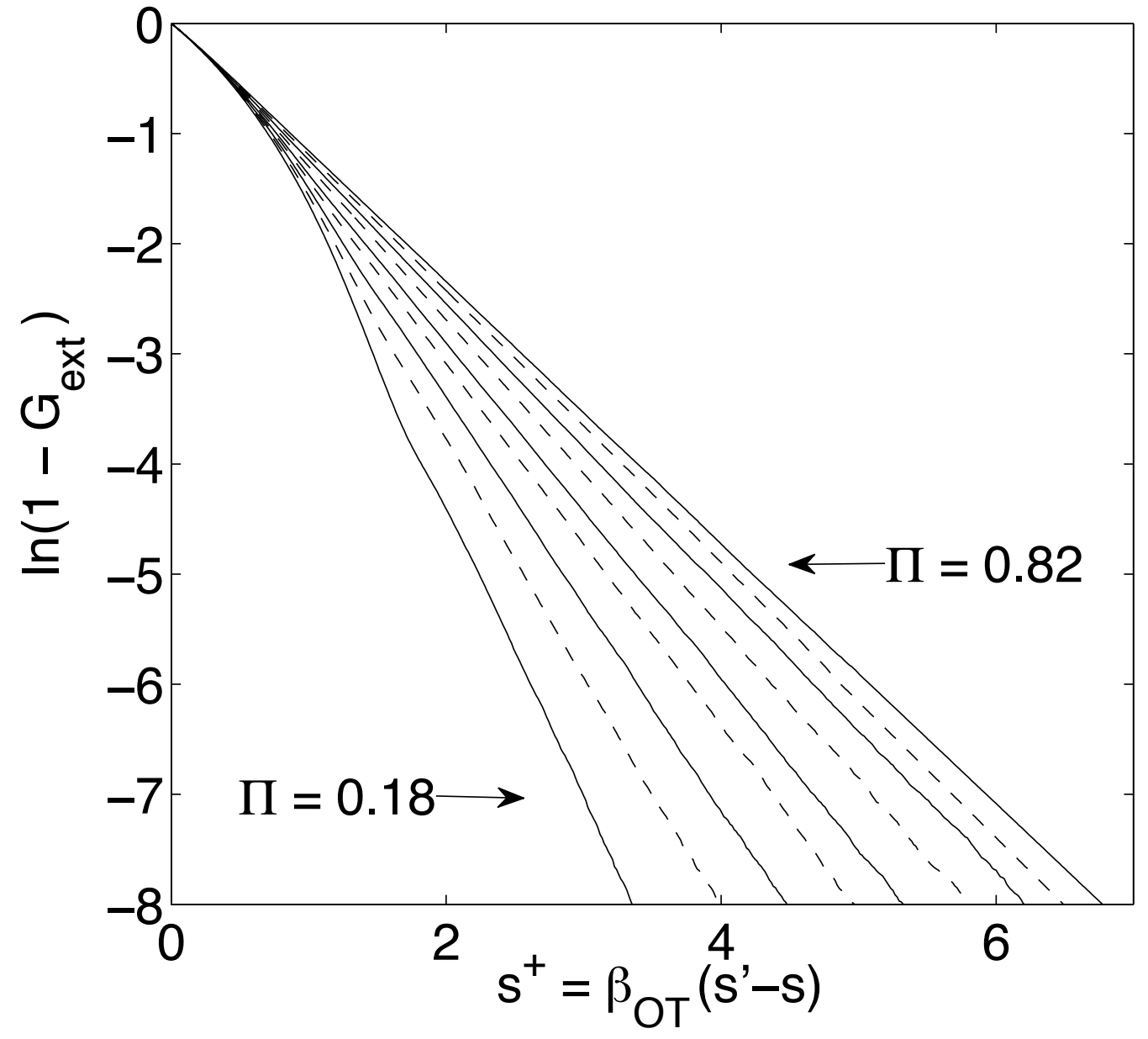




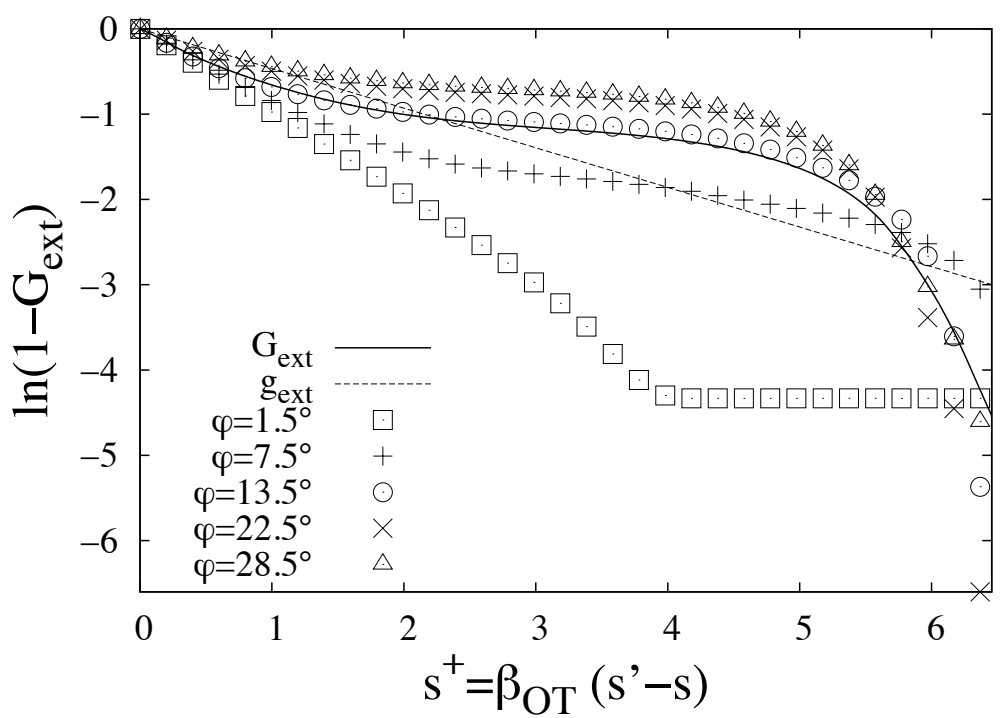

(a)

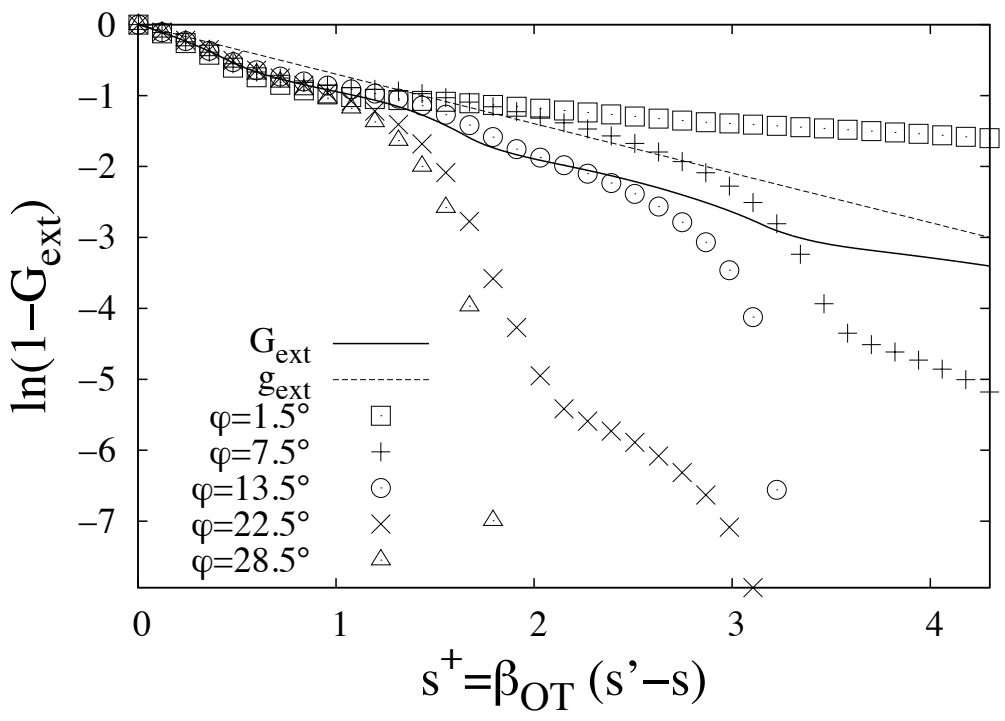

(b)

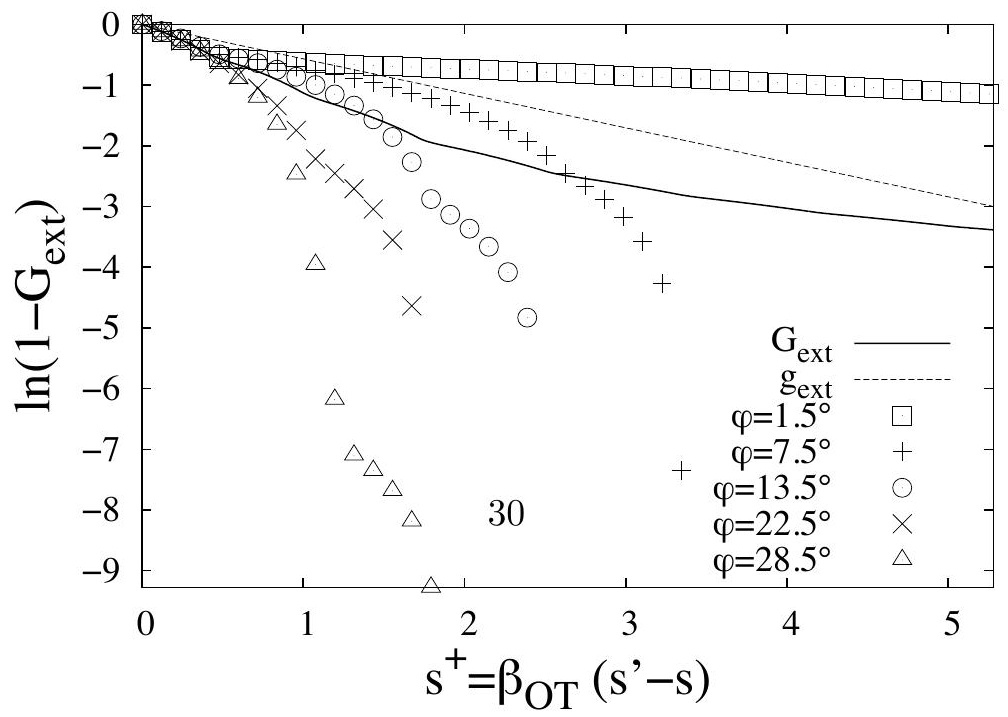



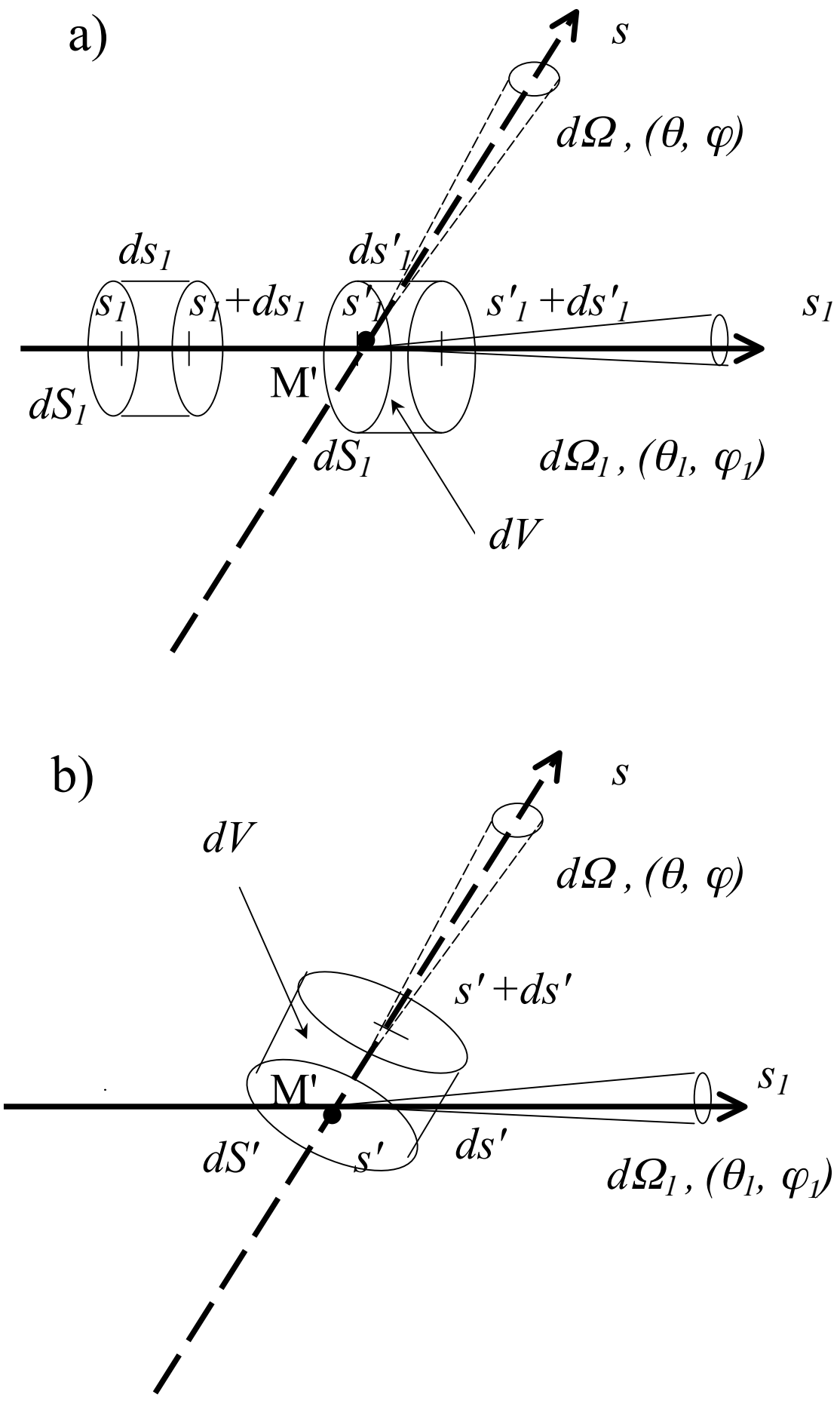


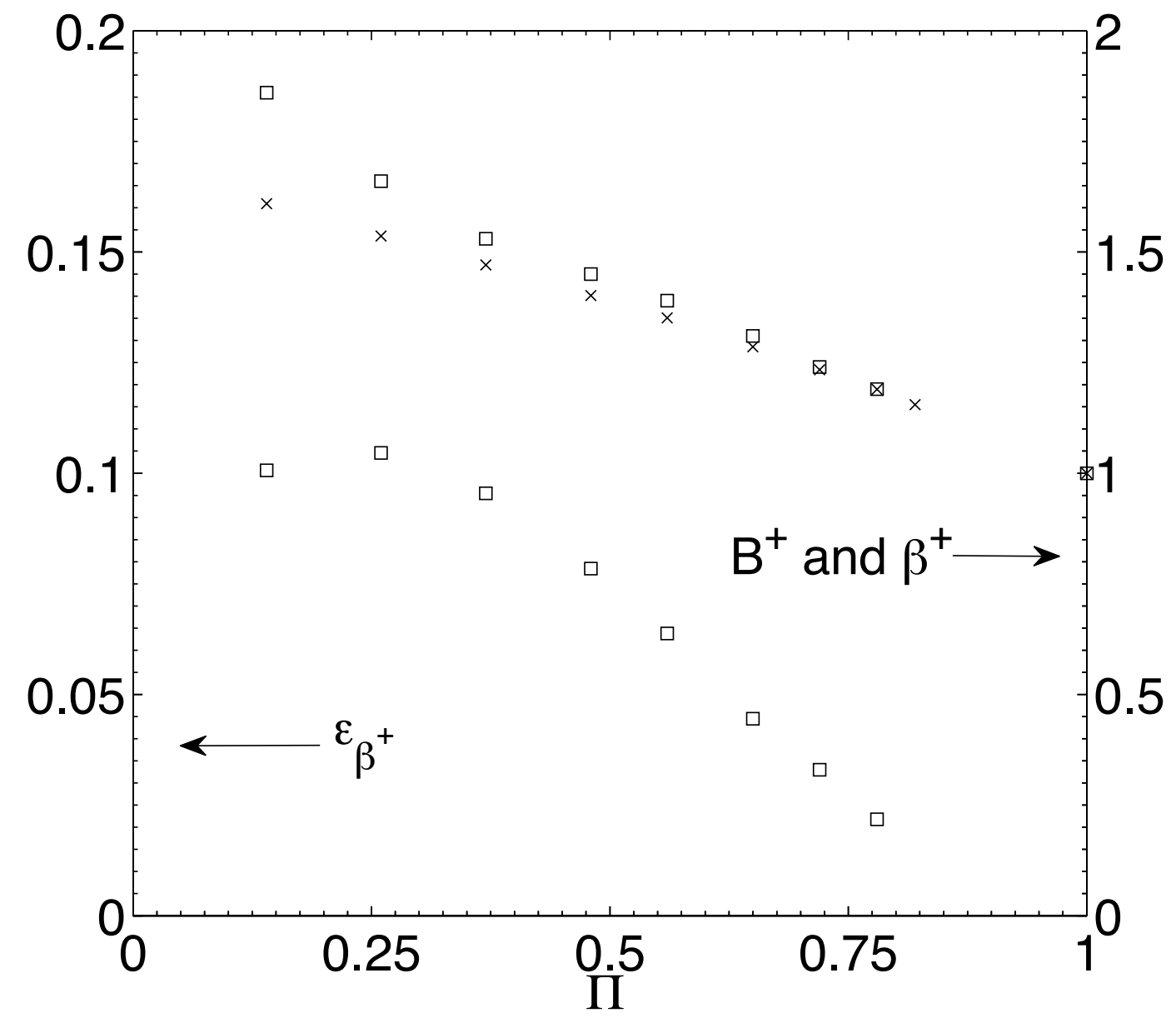




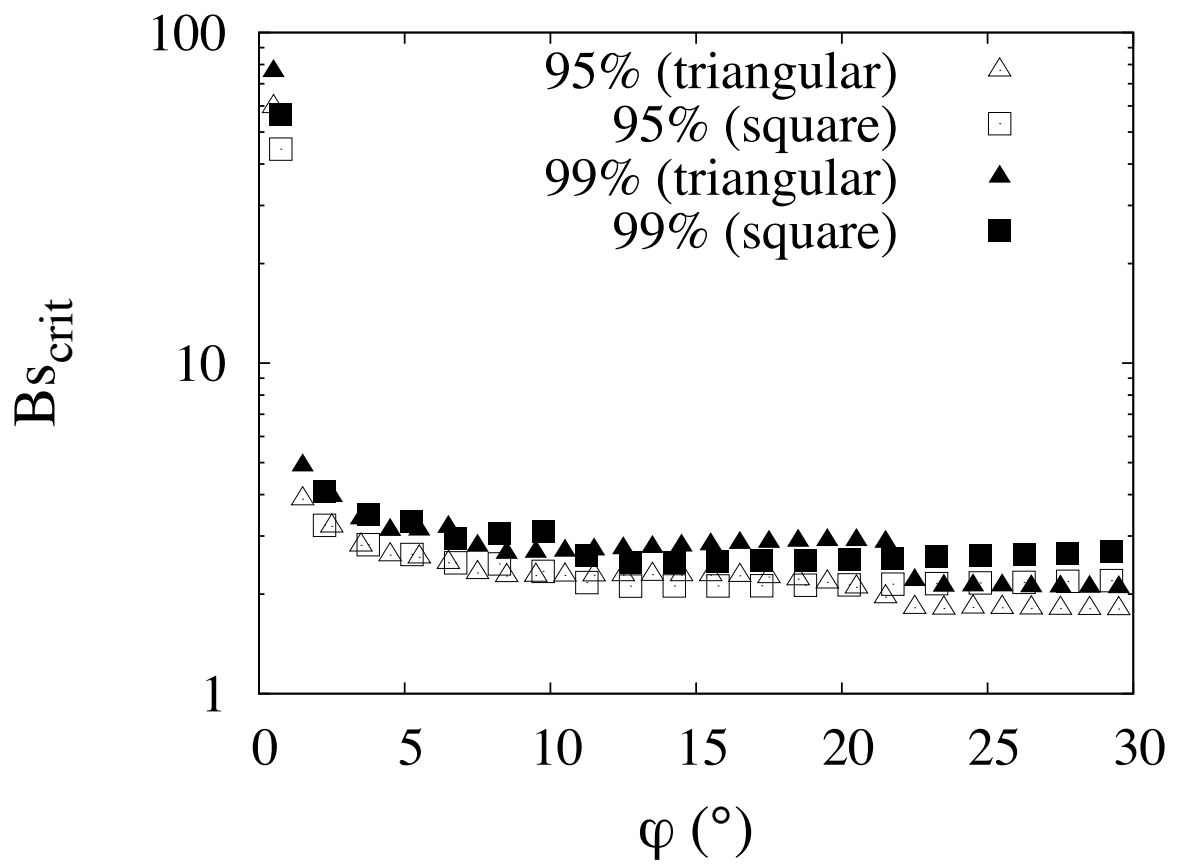

(a)

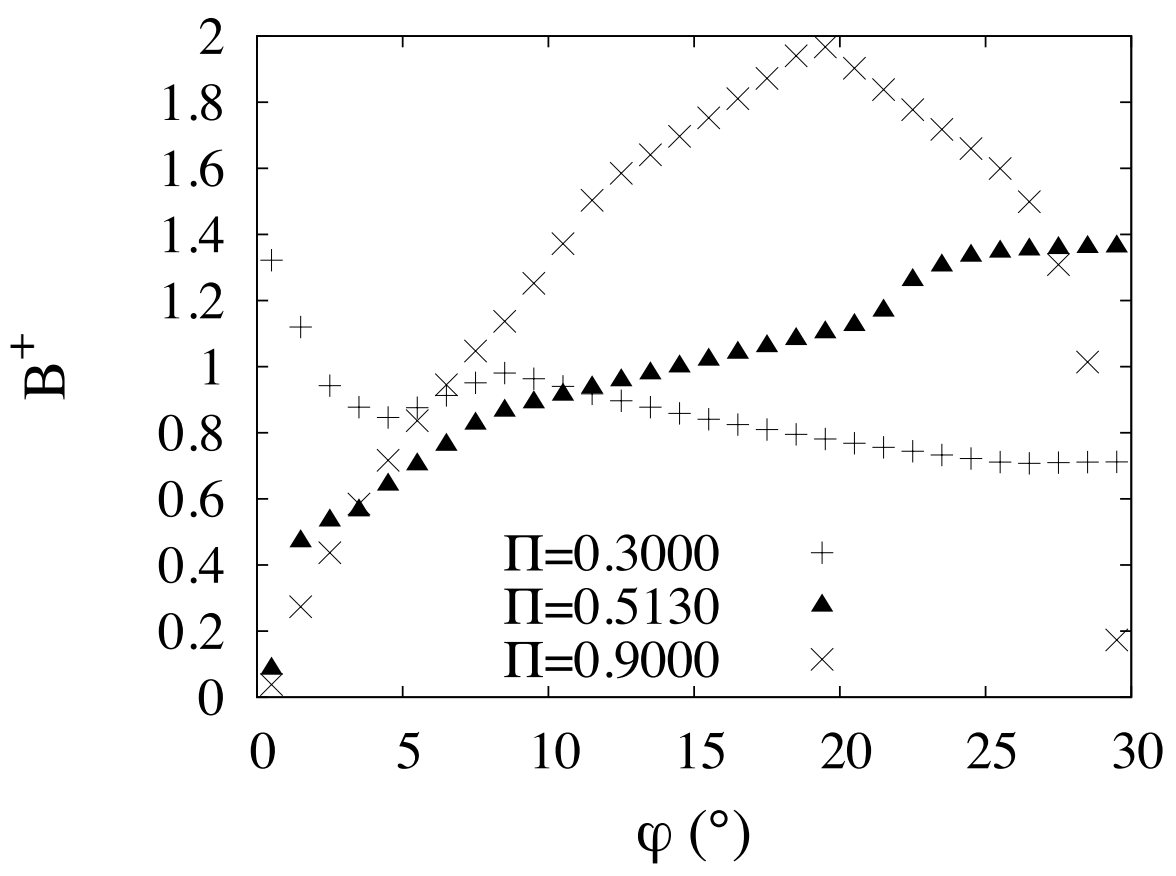

(b) 


\section{List of Tables}

$.1 \quad$ Non dimensional radiative conductivity $k^{+}$versus $\Pi$ and $\alpha^{h}$, for triangular and square configurations. . . . . . . . . . . . . . . . . 35 


\begin{tabular}{|c|c|c|c|c|c|c|c|c|c|c|c|}
\hline \multicolumn{3}{|c|}{$\alpha^{h}$} & $1 / 9$ & $2 / 9$ & $3 / 9$ & $4 / 9$ & $5 / 9$ & $6 / 9$ & $7 / 9$ & $8 / 9$ & 1 \\
\hline \multirow{29}{*}{$\Pi$} & \multirow{15}{*}{ Triangular } & 0.1500 & 16.93 & 17.61 & 18.35 & 19.17 & 20.06 & 21.06 & 22.17 & 23.41 & 24.81 \\
\hline & & 0.2147 & 8.43 & 8.78 & 9.16 & 9.57 & 10.03 & 10.54 & 11.10 & 11.73 & 12.44 \\
\hline & & 0.3000 & 4.83 & 5.04 & 5.26 & 5.51 & 5.78 & 6.07 & 6.40 & 6.77 & 7.19 \\
\hline & & 0.3760 & 5.06 & 5.25 & 5.45 & 5.68 & 5.93 & 6.20 & 6.50 & 6.84 & 7.23 \\
\hline & & 0.4100 & 7.46 & 7.67 & 7.89 & 8.13 & 8.40 & 8.70 & 9.03 & 9.39 & 9.80 \\
\hline & & 0.4460 & 12.09 & 12.31 & 12.56 & 12.83 & 13.13 & 13.45 & 13.81 & 14.22 & 14.67 \\
\hline & & 0.5130 & 25.80 & 26.07 & 26.37 & 26.70 & 27.06 & 27.45 & 27.89 & 28.37 & 28.92 \\
\hline & & 0.5630 & 39.14 & 39.45 & 39.78 & 40.15 & 40.55 & 41.00 & 41.49 & 42.04 & 42.65 \\
\hline & & 0.6000 & 50.18 & 50.52 & 50.88 & 51.27 & 51.71 & 52.18 & 52.71 & 53.30 & 53.96 \\
\hline & & 0.6500 & 65.69 & 66.05 & 66.45 & 66.88 & 67.35 & 67.87 & 68.44 & 69.08 & 69.80 \\
\hline & & 0.7000 & 80.74 & 81.13 & 81.55 & 82.01 & 82.51 & 83.06 & 83.67 & 84.35 & 85.11 \\
\hline & & 0.7500 & 94.91 & 95.32 & 95.76 & 96.24 & 96.76 & 97.34 & 97.97 & 98.68 & 99.47 \\
\hline & & 0.8000 & 107.03 & 107.46 & 107.91 & 108.41 & 108.96 & 109.56 & 110.22 & 110.95 & 111.77 \\
\hline & & 0.8500 & 115.63 & 116.09 & 116.58 & 117.11 & 117.69 & 118.33 & 119.03 & 119.81 & 120.68 \\
\hline & & 0.9000 & 119.37 & 119.85 & 120.36 & 120.91 & 121.52 & 122.18 & 122.92 & 123.73 & 124.64 \\
\hline & \multirow{14}{*}{ Square } & 0.2147 & 3.60 & 3.76 & 3.93 & 4.11 & 4.32 & 4.54 & 4.78 & 5.04 & 5.34 \\
\hline & & 0.3000 & 6.72 & 6.88 & 7.06 & 7.25 & 7.46 & 7.69 & 7.94 & 8.21 & 8.52 \\
\hline & & 0.3760 & 17.20 & 17.41 & 17.62 & 17.86 & 18.12 & 18.41 & 18.73 & 19.08 & 19.46 \\
\hline & & 0.4100 & 23.22 & 23.44 & 23.68 & 23.94 & 24.22 & 24.54 & 24.88 & 25.26 & 25.69 \\
\hline & & 0.4460 & 29.97 & 30.20 & 30.46 & 30.74 & 31.05 & 31.39 & 31.76 & 32.17 & 32.63 \\
\hline & & 0.5130 & 42.90 & 43.17 & 43.46 & 43.77 & 44.11 & 44.49 & 44.90 & 45.36 & 45.87 \\
\hline & & 0.5630 & 52.12 & 52.40 & 52.71 & 53.04 & 53.41 & 53.81 & 54.25 & 54.73 & 55.28 \\
\hline & & 0.6000 & 58.53 & 58.82 & 59.14 & 59.49 & 59.87 & 60.28 & 60.74 & 61.25 & 61.81 \\
\hline & & 0.6500 & 66.61 & 66.93 & 67.27 & 67.64 & 68.04 & 68.49 & 68.98 & 69.51 & 70.11 \\
\hline & & 0.7000 & 74.10 & 74.44 & 74.82 & 75.23 & 75.67 & 76.16 & 76.69 & 77.28 & 77.94 \\
\hline & & 0.7500 & 81.02 & 81.41 & 81.82 & 82.27 & 82.76 & 83.29 & 83.88 & 84.52 & 85.24 \\
\hline & & 0.8000 & 87.50 & 87.91 & 88.36 & 88.84 & 89.37 & 89.94 & 90.58 & 91.27 & 92.05 \\
\hline & & 0.8500 & 92.29 & 92.73 & 93.19 & 93.70 & 94.25 & 94.85 & 95.51 & 96.24 & 97.05 \\
\hline & & 0.9000 & 92.95 & 93.40 & 93.90 & 94.43 & 95.01 & 95.65 & 96.35 & 97.12 & 97.98 \\
\hline
\end{tabular}

